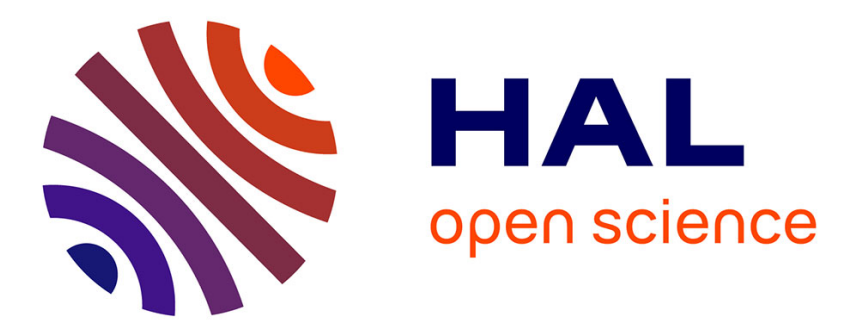

\title{
Simulation of complex impact problems with implicit time algorithms: Application to a turbo-engine blade loss problem
}

Ludovic Noels, Laurent Stainier, Jean-Philippe Ponthot

\section{To cite this version:}

Ludovic Noels, Laurent Stainier, Jean-Philippe Ponthot. Simulation of complex impact problems with implicit time algorithms: Application to a turbo-engine blade loss problem. International Journal of Impact Engineering, 2005, 32 (1-4), pp.358-386. 10.1016/j.ijimpeng.2005.06.003 . hal-01007295

\section{HAL Id: hal-01007295 \\ https://hal.science/hal-01007295}

Submitted on 10 Nov 2021

HAL is a multi-disciplinary open access archive for the deposit and dissemination of scientific research documents, whether they are published or not. The documents may come from teaching and research institutions in France or abroad, or from public or private research centers.
L'archive ouverte pluridisciplinaire HAL, est destinée au dépôt et à la diffusion de documents scientifiques de niveau recherche, publiés ou non, émanant des établissements d'enseignement et de recherche français ou étrangers, des laboratoires publics ou privés.

\section{(ㄷ)(1) $\$$}

Distributed under a Creative Commons Attribution - NonCommerciall 4.0 International 


\title{
Simulation of complex impact problems with implicit time algorithms: Application to a turbo-engine blade loss problem
}

\author{
L. Noels ${ }^{1}$, L. Stainier ${ }^{2}$, J.-P. Ponthot* \\ University of Liège, LTAS-Milieux Continus \& Thermomécanique, Chemin des Chevreuils 1, B-4000 Liège, Belgium
}

Recent developments, in non-linear structural dynamics, have led to a new kind of implicit algorithms: the energy-momentum conserving algorithm (EMCA) and the energy-dissipative, momentum-conserving algorithm. Contrarily to commonly used algorithms, such as the explicit central difference or the $\alpha$ generalized method, the stability of those algorithms is always ensured in the non-linear range. Thanks to this unconditional stability the only requirement on the time step size is that it must be small enough to capture the physics. This requirement is less restrictive than a conditional stability. In previous works, we have developed a new formulation of the internal forces for a hypoelastic model, that leads to an EMCA. In this paper, we will extend this formulation to an energy-dissipative, momentum-conserving algorithm. We will prove with an academic example, that our algorithm is more accurate than the $\alpha$-generalized method in the non-linear range. Then we will simulate a blade loss problem to demonstrate the efficiency of our developments on complex dynamics simulations.

Keywords: Energy-dissipative momentum conserving; Dynamics time integration; Hypoelastic models; Large strain; Finite-element simulation

\footnotetext{
*Corresponding author. Tel.: + 32436693 10; fax: + 3243669141.

E-mail address: jp.ponthot@ulg.ac.be (J.-P. Ponthot).

${ }^{1}$ Research Fellow at the Belgian National Fund for Scientific Research (FNRS).

${ }^{2}$ Research Associate at the Belgian National Fund for Scientific Research (FNRS).
} 


\section{Introduction}

When simulating impact problems, time integration of the equations off evolution occurs in the non-linear range. Usually, explicit algorithms are used in such a context. Nevertheless, due to its lack of stability in the non-linear range, and its limitation in time step size, an implicit scheme could advantageously be used. The most widely used implicit algorithm is the Newmark algorithm [1]. For linear models, this algorithm is unconditionally stable. For non-linear models, Belytschko and Schoeberle [2] proved that the discrete energy, computed from the work of the internal forces and from the kinetic energy, is bounded if it remains positive. Nevertheless, since the work of the internal forces is different from the internal energy variation when the Newmark algorithm is used in the non-linear range, Hughes et al. [3] have proved that the Newmark algorithm remains physically consistent only for small time step sizes. To avoid divergence due to the numerical instabilities, numerical damping was thus introduced, leading to the generalized- $\alpha$ methods [4]. Nevertheless, the unconditional stability of these methods occurs only for linear systems or asymptotically for the high frequency in the non-linear range [5].

Therefore, a new kind of implicit algorithm that remains stable in the non-linear range appeared. The first algorithm verifying these properties was described by Simo and Tarnow [6]. They called this algorithm energy-momentum conserving algorithm or EMCA. It consists in a mid-point scheme with an adequate evaluation of the internal forces. This adequate evaluation was given for a Saint Venant-Kirchhoff hyperelastic material. A generalization to other hyperelastic models was given by Gonzalez [7]. The EMCA was recently extended to dynamic finite deformation plasticity, with a hyperelastic formulation, by Meng and Laursen [8]. We have recently $[9,10]$ established a new expression of the internal forces for the hypoelastic materials using the final rotation scheme. When associated with the mid-point scheme, this expression ensures the conservation laws of mechanics for a hypoelastic constitutive model. Moreover, using the radial return mapping, we proved that this adaptation remains consistent with the Drucker postulate when plastic deformation occurs. Nevertheless, if the EMCA remains stable in the nonlinear range, the presence of high-frequency modes, resulting from the finite-element discretization, can lead to divergence of the Newton-Raphson iterations. Armero and Romero $[11,12]$ have introduced numerical dissipation in the conserving algorithms, for hyperelastic models. This dissipation only affects the total energy but preserves the angular momentum. Moreover, it is proved to be stable in the non-linear range, contrarily to the $\alpha$-generalized algorithms. It is called energy-dissipative momentum conserving algorithm or EDMC. The goal of this paper is to extend this EDMC algorithm to the treatment of finite plasticity. This algorithm was recently successfully extended to the treatment of contact interaction by the authors [13]. In that paper it is demonstrated that accuracy of contact interaction is ensured when dealing with large time step size (compared to the time step size of an explicit simulation). By combining this contact algorithm with the formulation of finite plasticity proposed in the present paper, we are able to treat complex impact problem with an implicit scheme. Let us note that the purpose of considering implicit schemes is to be able to treat this kind of simulation with a combined implicit/ explicit time integration algorithm. Such a combination can reduce the computational time without loss of accuracy [14-16]. Nevertheless it requires an efficient implicit time integration method in the non-linear range. This paper demonstrates that large strain/strain rate plasticity can be treated efficiently with the new implicit time integration algorithm. 
In this paper, we propose to introduce numerical dissipation in our hypoelastic conserving model. The plan is as follows: Section 2 will expose the preliminaries such as the dynamics conservation laws and the finite-element discretization. In Section 3, we will recall the EDMC algorithm principles. In Section 4, we will develop a forces formulation for a hypoelastic model that leads the integration algorithm to verify the energy dissipation and the conserving momentum properties. In Section 5, we will show the accuracy of our algorithm on the Taylor bar problem. We will also demonstrate the ability of theses developments to simulate the complex problem of a blade loss in a aero engine. Finally, we will draw some conclusions.

\section{Preliminaries}

In this section we will define the notations in use in this work. Therefore, we will be able to recall the continuum laws. Then we will introduce the finite element discretization.

\subsection{Notations}

Let $\mathbb{R} \subset \mathbb{R}^{3}$ be the manifold of the points defining the body and $\mathbb{S} \subset \mathbb{R}^{3}$ be the manifold of the boundary. We define two configurations: the initial configuration referred to by subscript 0 and the current configuration at time $t$. Let $\rho_{0}: \mathbb{V}_{0} \rightarrow \mathbb{R}_{+}$be the initial density. Boundary $\mathbb{S}$ is decomposed into two parts: the first one $\mathbb{S}_{\vec{x}}$ is the part where the displacements are known and the second one $\mathbb{S}_{\vec{T}}$ is the part where the surface tractions are known. It yields $\mathbb{S}_{\vec{x}} \cup \mathbb{S}_{\vec{T}}=\mathbb{S}$ and $\mathbb{S}_{\vec{x}} \cap \mathbb{S}_{\vec{T}}=0$. Let $\vec{x}$ be the current positions and $\vec{x}_{0}$ be the initial positions. Therefore, the two-point gradient of deformation tensor is defined by

$$
\mathbf{F} \equiv \frac{\partial \vec{x}}{\partial \vec{x}_{0}} \text { with } \mathbf{f} \equiv \mathbf{F}^{-1} \quad \text { and } \quad J \equiv \operatorname{det} \mathbf{F} .
$$

Conservation of the mass leads to

$$
\rho \mathrm{d} \mathbb{V}=\rho_{0} \mathrm{~d} \mathbb{V}_{0} \quad \text { and } \quad \rho J=\rho_{0} J_{0} .
$$

Let $\mathbb{X}$ be the manifold of the admissible positions

$$
\mathbb{X} \equiv\left\{\vec{x}: \mathbb{V}_{0} \rightarrow \mathbb{R}^{3} \mid\left[J>0 \text { and }\left.\vec{x}\right|_{\mathbb{S}_{\vec{x}}}=\overrightarrow{\vec{x}}\right] \quad \forall \vec{x}_{0} \in \mathbb{V}_{0}\right\}
$$

with $\overline{\vec{x}}$ the known positions. Let $t$ be the current time and let $\mathbb{T}=\left[0, t_{f}\right]$ be the integration interval. Therefore, the motion of the body is defined by $t \in \mathbb{T} \rightarrow \vec{x}(t) \in \mathbb{X}$. During this motion, the body is subject to specific loads $\vec{b}(t): \mathbb{V}_{0} \times \mathbb{T} \rightarrow \mathbb{R}^{3}$. Let $\boldsymbol{\Sigma}$ be the Cauchy stress tensor. Boundary pressures $\vec{T}_{S}(t): \mathbb{S}_{\vec{T} 0} \times \mathbb{T} \rightarrow \mathbb{R}^{3}$ lead to the condition $\vec{T}_{S}(t)=\Sigma(t) \vec{n}(t)$ with $\vec{n}$ the outward unit normal to $\mathbb{S}$.

The body is now decomposed into finite elements thanks to shape functions $\varphi^{\xi}: \mathbb{V}_{0} \rightarrow \mathbb{R}$ with $\xi \in[1, N]$ ( $N$ the total number of nodes), and with $\varphi^{\xi}\left(\vec{x}_{0}^{\mu}\right)=\delta_{\xi}^{\mu}$ ( $\delta$ is the Kronecker symbol). It leads for each node $\xi \in[1, N]$

$$
\vec{x}\left(\vec{x}_{0}\right)=\varphi^{\xi}\left(\vec{x}_{0}\right) \vec{x}^{\xi}, \dot{\vec{x}}\left(\vec{x}_{0}\right)=\varphi^{\xi}\left(\vec{x}_{0}\right) \dot{\vec{x}}^{\xi} \text { and } \ddot{\vec{x}}\left(\vec{x}_{0}\right)=\varphi^{\xi}\left(\vec{x}_{0}\right) \ddot{\vec{x}}^{\xi},
$$


where Einstein's notations are used. Let $\vec{v}$ be an admissible virtual displacement defined by the manifold

$$
\mathbb{D} \equiv\left\{\vec{v}: \mathbb{V}_{0} \rightarrow \mathbb{R}^{3} \mid\left[\left.\vec{v}\right|_{\mathbb{S}_{\vec{x}}}=0 \text { et } \vec{v}\left(\vec{x}_{0}, 0\right)=0, \vec{v}\left(\vec{x}_{0}, t_{f}\right)=0 \forall \vec{x}_{0} \in \mathbb{V}_{0}\right]\right\} .
$$

Let $\mathbb{D}^{v} \subset \mathbb{D}$ be the manifold of admissible virtual displacements $\delta \vec{x}$ that can be decomposed such as (4).

\subsection{The continuum dynamics}

The following quasi-variational principle (principle of virtual power of forces) must hold $\forall \delta \vec{x} \in \mathbb{D}^{v}[17$, p. 412]:

$$
\int_{0}^{t_{f}}\left\{\int_{\mathbb{V}}\left[\rho \ddot{\vec{x}} \cdot \delta \vec{x}+\Sigma^{\mathrm{T}}: \frac{\partial \delta \vec{x}}{\partial \vec{x}}-\rho \vec{b} \cdot \delta \vec{x}\right] \mathrm{d} \mathbb{V}-\int_{\mathbb{S}_{\vec{T}}}\left[\vec{T}_{S} \cdot \delta \vec{x}\right] \mathrm{d} \mathbb{S}\right\} \mathrm{d} t=0 .
$$

Integrating by parts, one gets

$$
\begin{aligned}
& \underbrace{\int_{\mathbb{V}}\{\rho \ddot{\vec{x}} \cdot \delta \vec{x}\} \mathrm{d} \mathbb{V}}_{\equiv \delta K}=\underbrace{\int_{\mathbb{V}}\{\rho \vec{b} \cdot \delta \vec{x}\} \mathrm{d} \mathbb{V}+\int_{\mathbb{S}_{\vec{T}}}\left\{\vec{T}_{S} \cdot \delta \vec{x}\right\} \mathrm{d} \mathbb{S}}_{\equiv \delta W_{\mathrm{ext}}} \\
& -\underbrace{\int_{\mathbb{V}}\left\{\Sigma^{\mathrm{T}}: \frac{\partial \delta \vec{x}}{\partial \vec{x}}\right\} \mathrm{d} \mathbb{V}}_{\equiv \delta W_{\text {int }}} \quad \forall t \in \mathbb{T}
\end{aligned}
$$

with $\delta W_{\text {int }}, \delta W_{\text {ext }}$ and $\delta K$, respectively, the virtual work of internal forces, the virtual work of external forces and the virtual work of inertia forces. This principle leads to the dynamics conservation laws.

\subsubsection{Conservation of linear momentum}

Let $\vec{L}$ be the linear momentum defined by

$$
\vec{L} \equiv \int_{\mathbb{V}}\{\rho \dot{\vec{x}}\} \mathrm{d} \mathbb{V}=\int_{\mathbb{V}_{0}}\left\{\rho_{0} \dot{\vec{x}}\right\} \mathrm{d} \mathbb{V}_{0},
$$

where Eq. (2) has been used. If $\delta \vec{x} \in \mathbb{D}^{v}$ is taken constant (rigid body translation), Eq. (7) leads to the conservation of the linear momentum

$$
\dot{\vec{L}}=\underbrace{\int_{\mathbb{V}}\{\rho \vec{b}\} \mathrm{d} \mathbb{V}+\int_{\mathbb{S}_{\vec{T}}}\left\{\vec{T}_{S}\right\} \mathrm{d} \mathbb{S}}_{\equiv \vec{F}_{\text {ext }}} \forall t \in \mathbb{T} .
$$

\subsubsection{Conservation of angular momentum}

Let $\vec{J}$ be the angular momentum defined by

$$
\vec{J} \equiv \int_{\mathbb{V}}\{\rho \vec{x} \wedge \dot{\vec{x}}\} \mathrm{d} \mathbb{V}=\int_{\mathbb{V}_{0}}\left\{\rho_{0} \vec{x} \wedge \dot{\vec{x}}\right\} \mathrm{d} \mathbb{V}_{0}
$$


Taking $\delta \vec{x}=\vec{\eta} \wedge \vec{x}$ with $\vec{\eta}$ constant (rigid body rotation), since $\boldsymbol{\Sigma}$ is symmetric, and $\vec{\eta}$ is an arbitrary constant, Eq. (7) leads to the conservation of the angular momentum

$$
\dot{\vec{J}}=\int_{\mathbb{V}}\{\rho \vec{x} \wedge \vec{b}\} \mathrm{d} \mathbb{V}+\int_{\mathbb{S}_{\vec{T}}}\left\{\vec{x} \wedge \vec{T}_{S}\right\} \mathrm{d} \mathbb{S} \quad \forall t \in \mathbb{T} .
$$

\subsubsection{Conservation of the energy}

Let $K, W_{\text {int }}$ and $W_{\text {ext }}$ be, respectively, the kinetic energy, the internal forces work and the external forces work, with

$$
\begin{aligned}
& K \equiv \int_{\mathbb{V}}\left\{\frac{1}{2} \rho \dot{\vec{x}}^{2}\right\} \mathrm{d} \mathbb{V}=\int_{\mathbb{V}_{0}}\left\{\frac{1}{2} \rho_{0} \dot{\vec{x}}^{2}\right\} \mathrm{d} \mathbb{V}_{0}, \\
& \dot{W}_{\text {int }} \equiv \int_{\mathbb{V}}\left\{\boldsymbol{\Sigma}^{\mathrm{T}}:[\dot{\mathbf{F}} \mathbf{f}]\right\} \mathrm{d} \mathbb{V}, \\
& \dot{W}_{\text {ext }} \equiv \int_{\mathbb{V}}\{\rho \vec{b} \cdot \dot{\vec{x}}\} \mathrm{d} \mathbb{V}+\int_{\mathbb{S}_{\vec{T}}}\left\{\vec{T}_{S} \cdot \dot{\vec{x}}\right\} \mathrm{d} \mathbb{S},
\end{aligned}
$$

where Eq. (2) is used. If the internal forces power $\dot{W}_{\text {int }}$ is decomposed into a reversible part $\dot{U}_{\text {int }}$ and an irreversible part $\dot{\Delta}_{\text {int }} \geqslant 0$ (plastic dissipation, etc.) and if $E$ is the system energy, one gets

$$
\dot{W}_{\text {int }} \equiv \dot{U}_{\text {int }}+\dot{\Delta}_{\text {int }} \text { and } E \equiv K+U_{\text {int }} .
$$

Therefore, if $\delta \vec{x}=\dot{\vec{x}}$, Eq. (7) leads to the first thermodynamics principle

$$
\dot{E}=\dot{W}_{\text {ext }}-\dot{\Delta}_{\text {int }} \quad \forall t \in \mathbb{T} \text {. }
$$

\subsection{Finite-element decomposition}

Thanks to Eq. (2) and to Eq. (4), the discrete terms of Eq. (7) can be rewritten such that

$$
\begin{aligned}
& \delta K=\int_{\mathbb{V}_{0}}\left\{\rho_{0} \varphi^{\xi} \varphi^{\mu}\right\} \mathrm{d} \mathbb{V}_{0}[\ddot{\vec{x}}]^{\mu} \cdot \delta \vec{x}^{\xi}=M^{\xi \mu}[\ddot{\vec{x}}]^{\mu} \cdot \delta \vec{x}^{\xi}, \\
& \delta W_{\text {ext }}=\int_{\mathbb{V}_{0}}\left\{\rho_{0} \vec{b} \varphi^{\xi}\right\} \mathrm{d} \mathbb{V}_{0} \cdot \delta \vec{x}^{\xi}+\int_{\mathbb{S}_{\vec{T}}}\left\{\vec{T}_{S} \varphi^{\xi}\right\} \mathrm{d} \mathbb{S} \cdot \delta \vec{x}^{\xi}=\left[\vec{F}_{\text {ext }}\right]^{\xi} \cdot \delta \vec{x}^{\xi}, \\
& \delta W_{\text {int }}=\int_{\mathbb{V}_{0}}\left\{\Sigma^{\mathrm{T}}\left[\frac{\partial \varphi^{\xi}}{\partial \vec{x}}\right]^{\mathrm{T}} J\right\} \mathrm{d} \mathbb{V}_{0} \cdot \delta \vec{x}^{\xi}=\underbrace{\int_{\mathbb{V}_{0}}\left\{\Sigma^{\mathrm{T}} \mathbf{f}^{\mathrm{T}} \vec{D}^{\xi} J\right\} \mathrm{d} \mathbb{V}_{0} \cdot \delta \vec{x}^{\xi},}_{\equiv \vec{F}_{\text {int }}^{\xi}}
\end{aligned}
$$

where $M^{\xi \mu}$ is the mass related to nodes $\xi$ and $\mu$ and where $\vec{D}$ is the derivative value, in the initial configuration, of the shape functions (i.e., $\vec{D}^{\xi}=\partial \varphi^{\xi} / \partial \vec{x}_{0}$ ). Since $\delta \vec{x} \in \mathbb{D}^{v}$ is an arbitrary vector, Eq. (7) leads to the balance equation

$$
M^{\xi \mu}[\ddot{\vec{x}}]^{\mu}=\left[\vec{F}_{\text {ext }}-\vec{F}_{\text {int }}\right]^{\xi} \quad \forall t \in \mathbb{T} .
$$


To be able to integrate this relation in time, $\mathbb{T}$ is decomposed into some intervals $\left[t^{n}, t^{n+1}\right]$ such that $\mathbb{T}=\bigcup_{n=0}^{n=n^{f}}\left[t^{n}, t^{n+1}\right]$. Let $\Delta t=t^{n+1}-t^{n}$ be the time step size. Superscripts $n$ and $n+1$ will refer to configurations in time $t^{n}$ and $t^{n+1}$. To be physically consistent, the integration scheme must verify Eq. (9), Eqs. (11) and (14).

\section{The energy-dissipative momentum conserving algorithm}

Once the balance Eq. (16) is established for all times $t$, this relation must be integrated in time. To achieve this goal, Armero and Romero [11,12] have introduced velocities dissipation $\vec{G}_{\text {diss }}$ and forces dissipation $\vec{F}_{\text {diss }}$ in Simo and Tarnow EMCA scheme. Both vectors $\vec{G}_{\text {diss }}$ and $\vec{F}_{\text {diss }}$ must be considered simultaneously to avoid bifurcation in the spectral analysis of the amplification matrix. In this section we will present the headlines of the EDMC algorithm. Then we will propose a predictor-corrector algorithm to solve the set of equations. Next, we will deduce the conditions on the forces to verify the conservations laws expressed by Eqs. (9), (11) and (14).

\subsection{Description}

The relation between positions and velocities at node $\xi$ becomes

$$
\left[\vec{x}^{n+1}\right]^{\xi}=\left[\vec{x}^{n}\right]^{\xi}+\frac{\Delta t}{2}\left[\dot{\vec{x}}^{n+1}\right]^{\xi}+\frac{\Delta t}{2}\left[\dot{\vec{x}}^{n}\right]^{\xi}+\Delta t\left[\vec{G}_{\mathrm{diss}}^{n+1 / 2}\right]^{\xi} .
$$

This relation is a second-order approximation (in $\Delta t$ ) if $\vec{G}_{\text {diss }}=\mathcal{O}\left(\Delta t^{2}\right)$ and is a first-order approximation if $\vec{G}_{\text {diss }}=\mathscr{O}(\Delta t)$. A second-order approximation of the relations between the velocities and the accelerations at node $\xi$ is

$$
\left[\dot{\vec{x}}^{n+1}\right]^{\xi}=\left[\dot{\vec{x}}^{n}\right]^{\xi}+\frac{\Delta t}{2}\left[\ddot{\vec{x}}^{n+1}\right]^{\xi}+\frac{\Delta t}{2}\left[\ddot{\vec{x}}^{n}\right]^{\xi} .
$$

The balance Eq. (16) is discretized in time at node $\xi$ by

$$
\frac{1}{2} M^{\xi}\left[\ddot{\vec{x}}^{n+1}+\ddot{\ddot{x}}^{n}\right]^{\mu}=\left[\vec{F}_{\text {ext }}^{n+1 / 2}-\vec{F}_{\text {int }}^{n+1 / 2}-\vec{F}_{\text {diss }}^{n+1 / 2}\right]^{\xi} \text {. }
$$

This relation is a second-order approximation of Eq. (16) if $\vec{F}_{\text {diss }}=\mathcal{O}\left(\Delta t^{2}\right)$ and if the internal forces $\vec{F}_{\text {int }}^{n+1 / 2}$ are a second-order approximation of $\vec{F}_{\text {int }}\left(t^{n+1 / 2}\right)$. The set of Eqs. (17)-(19) is solved by a predictor-corrector algorithm. Prediction values are deduced from Eqs. (17) and (18) by taking $\ddot{\vec{x}}^{n+1}=0$

$$
\begin{aligned}
& {\left[\vec{x}^{n+1,0}\right]^{\xi}=\left[\vec{x}^{n}+\Delta t \dot{\vec{x}}^{n}+\frac{\Delta t^{2}}{4} \ddot{\vec{x}}^{n}+\Delta t \vec{G}_{\mathrm{diss}}^{n+\frac{1}{2}, 0}\right]^{\xi},} \\
& {\left[\dot{\vec{x}}^{n+1,0}\right]^{\xi}=\left[\dot{\vec{x}}^{n}+\frac{\Delta t}{2} \ddot{\vec{x}}^{n}\right]^{\xi} .}
\end{aligned}
$$


Linearization with the accelerations of Eq. (19) evaluated at Newton-Raphson iteration $i$, leads to

$$
\begin{aligned}
{\left[\mathbf{S}^{i}\right]^{\xi}\left[\Delta \ddot{\vec{x}}^{i+1}\right]^{\mu}=} & -\frac{1}{2} M^{\xi \mu}\left[\ddot{\vec{x}}^{n+1, i}+\ddot{\vec{x}}^{n}\right]^{\mu} \\
& -\left[\vec{F}_{\text {int }}^{n+1 / 2}\left(\vec{x}^{n+1, i}\right)+\vec{F}_{\text {diss }}^{n+1 / 2}\left(\vec{x}^{n+1, i}\right)-\vec{F}_{\mathrm{ext}}^{n+1 / 2}\left(\vec{x}^{n+1, i}\right)\right]^{\xi}
\end{aligned}
$$

with $\mathbf{S}$ the Jacobian matrix

$$
\begin{aligned}
\mathbf{S}^{\xi \mu} & =\frac{\partial\left\{\frac{1}{2} M^{\xi \nu}\left[\ddot{\vec{x}}^{n+1}+\ddot{\vec{x}}^{n}\right]^{v}+\left[\vec{F}_{\text {int }}^{n+1 / 2}+\vec{F}_{\mathrm{diss}}^{n+1 / 2}-\vec{F}_{\mathrm{ext}}^{n+1 / 2}\right]^{\xi}\right\}}{\partial\left[\ddot{\vec{x}}^{n+1}\right]^{\mu}} \\
& =\mathbf{K}^{\xi v} \frac{\partial \vec{x}^{v}}{\partial \ddot{\vec{x}}^{\mu}}+\frac{1}{2} M^{\xi \mu} \mathbf{I}=\mathbf{K}^{\xi \mu}\left[\frac{\Delta t^{2}}{4} \mathbf{I}+\frac{\Delta t^{2}}{2} \mathbf{G}\left(\dot{\vec{x}}^{\mu}\right)\right]+\frac{1}{2} M^{\xi \mu} \mathbf{I} .
\end{aligned}
$$

In this last expression, $\mathbf{K}^{\xi \mu}$ is the stiffness matrix and $\mathbf{G}$ is the derivative of $\vec{G}_{\text {diss }}$ with respect to the velocities

$$
\mathbf{K}^{\xi \mu}=\frac{\partial\left[\vec{F}_{\text {int }}^{n+1 / 2}+\vec{F}_{\text {diss }}^{n+1 / 2}-\vec{F}_{\text {ext }}^{n+1 / 2}\right]^{\xi}}{\partial\left[\vec{x}^{n+1}\right]^{\mu}} \text { and } \mathbf{G}\left(\dot{\vec{x}}^{\xi}\right)=\frac{\partial \vec{G}_{\mathrm{diss}}^{n+1 / 2}\left(\dot{\vec{x}}^{\xi}\right)}{\partial \dot{\vec{x}}^{\xi}} \text {. }
$$

Let us note that solving Eqs. (21) using $\Delta \ddot{\vec{x}}$ and not $\Delta \vec{x}$ avoids the inversion of matrix G. Details to obtain the Jacobian matrix are reported in the appendix. Finally, linearization with the accelerations of Eqs. (17) and (18) leads to correction values at iteration $i+1$ and at configuration $n+1$

$$
\begin{aligned}
{\left[\ddot{\vec{x}}^{n+1, i+1}\right]^{\mu}=} & {\left[\ddot{\vec{x}}^{n+1, i}+\alpha_{1 \mathrm{~s}} \Delta \ddot{\vec{x}}^{i+1}\right]^{\mu}, } \\
{\left[\dot{\vec{x}}^{n+1, i+1}\right]^{\mu}=} & {\left[\dot{\vec{x}}^{n+1, i}+\frac{\Delta t}{2} \alpha_{1 \mathrm{~s}} \Delta \ddot{x}^{i+1}\right]^{\mu}, } \\
{\left[\vec{x}^{n+1, i+1}\right]^{\mu}=} & {\left[\vec{x}^{n+1, i}+\alpha_{\mathrm{ls}} \frac{\Delta t^{2}}{4} \Delta \ddot{\vec{x}}^{i+1}\right]^{\mu} } \\
& +\Delta t\left[\vec{G}_{\mathrm{diss}}\left(\dot{x}^{n+1, i+1}\right)-\vec{G}_{\mathrm{diss}}\left(\dot{\vec{x}}^{n+1, i}\right)\right]^{\mu}
\end{aligned}
$$

with $\alpha_{\text {ls }}$ a line search parameter that enhances the Newton-Raphson resolution [18, p. 254].

\subsection{Verification of conservation laws}

In this section we will verify the conservation laws defined by Eqs. (9), (11) and (14).

\subsubsection{Conservation of linear momentum}

A sum on $\xi$ in Eq. (19) and the use of Eq. (18) leads to

$$
\underbrace{\sum_{\xi} M^{\xi \mu}\left[\dot{\vec{x}}^{n+1}\right]^{\mu}}_{\vec{L}^{n+1}}-\underbrace{\sum_{\xi} M^{\xi}\left[\dot{\vec{x}}^{n}\right]^{\mu}}_{\vec{L}^{n}}=\Delta t \sum_{\xi}\left[\vec{F}_{\mathrm{ext}}^{n+1 / 2}-\vec{F}_{\mathrm{int}}^{n+1 / 2}-\vec{F}_{\mathrm{diss}}^{n+1 / 2}\right]^{\xi} \text {, }
$$


where the continuous linear momentum $\vec{L}$ defined by Eq. (8) is discretized thanks to Eq. (4) in $\vec{L}=\sum_{\xi} M^{\xi} \mu \dot{\vec{x}}^{\mu}$. Eq. (25) is a discretization of Eq. (9) if

$$
\sum_{\xi}\left[\vec{F}_{\text {int }}^{n+1 / 2}\right]^{\xi}=0 \text { and } \sum_{\xi}\left[\vec{F}_{\text {diss }}^{n+1 / 2}\right]^{\xi}=0 .
$$

These two conditions must be simultaneously verified since the dissipation forces are independent of the internal forces.

\subsubsection{Conservation of angular momentum}

Thanks to Eqs. (17) and Eq. (18), the vector product between $\vec{x}^{n+1 / 2}=\left(\vec{x}^{n}+\vec{x}^{n+1}\right) / 2$ and Eq. (19) leads to

$$
\begin{aligned}
& \frac{1}{\Delta t} \underbrace{M^{\xi \mu}\left[\vec{x}^{n+1}\right]^{\xi} \wedge\left[\dot{\vec{x}}^{n+1}\right]^{\mu}}_{\vec{J}^{n+1}}-\frac{1}{\Delta t} \underbrace{M^{\xi \mu}\left[\vec{x}^{n}\right]^{\xi} \wedge\left[\dot{\vec{x}}^{n}\right]^{\mu}}_{\vec{J}^{n}} \\
& \quad=M^{\xi \mu}\left[\vec{G}_{\mathrm{diss}}^{n+1 / 2}\right]^{\xi} \wedge\left[\frac{\dot{\vec{x}}^{n+1}+\dot{\vec{x}}^{n}}{2}\right]^{\mu}+\left[\vec{x}^{n+1 / 2}\right]^{\xi} \wedge\left[\vec{F}_{\mathrm{ext}}^{n+1 / 2}-\vec{F}_{\mathrm{int}}^{n+1 / 2}-\vec{F}_{\mathrm{diss}}^{n+1 / 2}\right]^{\xi},
\end{aligned}
$$

where the continuous angular momentum $\vec{J}$ defined by Eq. (10) is discretized thanks to Eq. (4) in $\vec{J}=M^{\xi \mu} \vec{x}^{\xi} \wedge \dot{\vec{x}}^{\mu}$. Therefore, Eq. (27) is a discretization of (10) if

$$
\left[\frac{\vec{x}^{n+1}+\vec{x}^{n}}{2}\right]^{\xi} \wedge\left[\vec{F}_{\text {int }}^{n+1 / 2}\right]^{\xi}=0 \text { and }\left[\frac{\vec{x}^{n+1}+\vec{x}^{n}}{2}\right]^{\xi} \wedge\left[\vec{F}_{\text {diss }}^{n+1 / 2}\right]^{\xi}=0
$$

and if

$$
M^{\xi} \mu\left[\vec{G}_{\mathrm{diss}}^{n+1 / 2}\right]^{\xi} \wedge\left[\frac{\dot{\vec{x}}^{n+1}+\dot{\vec{x}}^{n}}{2}\right]^{\mu}=0 .
$$

This last expression allows the numerical dissipation to conserve the angular momentum.

\subsubsection{Conservation of energy}

Thanks to Eqs. (17) and (18), the dot product between

$$
\dot{\vec{x}}^{n+1 / 2}=\frac{\dot{\vec{x}}^{n}+\dot{\vec{x}}^{n+1}}{2}+\vec{G}_{\mathrm{diss}}^{n+1 / 2}
$$

and Eq. (19) leads to

$$
\begin{gathered}
\underbrace{\frac{M^{\xi \mu}}{2}\left[\dot{\vec{x}}^{n+1}\right]^{\xi} \cdot\left[\dot{\vec{x}}^{n+1}\right]^{\mu}}_{K^{n+1}}-\underbrace{\frac{M^{\xi} \mu}{2}\left[\dot{\vec{x}}^{n}\right]^{\xi} \cdot\left[\dot{\vec{x}}^{n}\right]^{\mu}}_{K^{n}}+M^{\xi \mu}\left[\dot{\vec{x}}^{n+1}-\dot{\vec{x}}^{n}\right]^{\mu} \cdot\left[\vec{G}_{\mathrm{diss}}^{n+1}\right]^{\xi} \\
=\underbrace{\left[\vec{x}^{n+1}-\vec{x}^{n}\right]^{\xi} \cdot\left[\vec{F}_{\mathrm{ext}}^{n+1 / 2}\right]^{\xi}}_{W_{\mathrm{ext}}^{n+1}-W_{\mathrm{ext}}^{n}}-\left[\vec{x}^{n+1}-\vec{x}^{n}\right]^{\xi} \cdot\left[\vec{F}_{\mathrm{int}}^{n+1 / 2}+\vec{F}_{\mathrm{diss}}^{n+1 / 2}\right]^{\xi},
\end{gathered}
$$


where the continuous kinetic energy $K$ defined in Eq. (12) is dicretized thanks to Eq. (4) in $K=\frac{1}{2} M^{\xi \mu} \dot{\vec{x}}^{\xi} \cdot \dot{\vec{x}}^{\mu}$ and where the power of the external forces $\dot{W}_{\text {ext }}$ defined in Eq. (12) is discretized and integrated in $W_{\mathrm{ext}}^{n+1}-W_{\mathrm{ext}}^{n}=\left[\vec{x}^{n+1}-\vec{x}^{n}\right]^{\xi} \cdot\left[\vec{F}_{\mathrm{ext}}^{n+1 / 2}\right]^{\xi}$. Let $E$ be the discretized energy, let $U_{\text {int }}$ be the discretized internal energy, let $W_{\text {int }}$ be the discretized work of the internal forces and let $\Delta_{\text {int }} \geqslant 0$ be the discretized internal dissipation during the step, all such that their continuous values are defined in Eq. (13). Therefore Eq. (14) can be discretized into

$$
E^{n+1}-E^{n}=W_{\mathrm{ext}}^{n+1}-W_{\mathrm{ext}}^{n}-\Delta_{\mathrm{int}}-\Delta_{\mathrm{num}},
$$

where $\Delta_{\text {num }} \geqslant 0$ is the numerical dissipation during the step. If this last expression is compared with Eq. (30), the internal forces must lead to

$$
\left[\vec{F}_{\mathrm{int}}^{n+1 / 2}\right]^{\xi} \cdot\left[\vec{x}^{n+1}-\vec{x}^{n}\right]^{\xi}=U_{\mathrm{int}}^{n+1}-U_{\mathrm{int}}^{n}+\Delta_{\mathrm{int}}
$$

and the dissipation values must lead to

$$
\underbrace{M^{\xi} \mu\left[\dot{\vec{x}}^{n+1}-\dot{\vec{x}}^{n}\right]^{\mu} \cdot\left[\vec{G}_{\text {diss }}^{n+1 / 2}\right]^{\xi}}_{\equiv \Delta_{K}}+\underbrace{\left[\vec{F}_{\text {diss }}^{n+1 / 2}\right]^{\xi} \cdot\left[\vec{x}^{n+1}-\vec{x}^{n}\right]^{\xi}}_{\equiv \Delta_{W}}=\Delta_{\text {num }} \geqslant 0 .
$$

The problem of the EDMC algorithm is to find a physically consistent expression of the internal forces and of the dissipation terms that verify Eqs. (26)-(28), (29), (32) and (33). In a previous work $[9,10]$, we have developed a new expression of the internal forces, for an elasto-plastic hypoelatic model, that verifies this conditions in the absence of numerical dissipation. The goal of this paper is to establish the expression of the dissipation forces for such a model. It will be achieved in the following section.

\section{Expression of the forces for a hypoelastic model}

In this section, we will establish the internal and dissipation forces expressions and the dissipation velocities expression for an elasto-plastic hypoelastic model. First, we will recall the hypoelastic model. Next, we will briefly expose the formulation of the internal forces we have established in $[9,10]$. Then we will be able to extend this theory to the formulation of the dissipation forces for such a model. The velocities dissipation will therefore be given. Finally, the spectral analysis will prove that the high frequencies are numerically dissipated.

\subsection{The hypoelastic model}

The two-point-deformation map $\mathbf{F}$ defined in Eq. (1) can be evaluated between configurations $m$ and $n$

$$
\mathbf{F}_{m}^{n} \equiv \frac{\partial \vec{x}^{n}}{\partial \vec{x}^{m}} \text { with } \mathbf{F}_{0}^{n}=\mathbf{F}_{m}^{n} \mathbf{F}_{0}^{m}
$$


Thanks to the Polar Decomposition theorem, this tensor can be decomposed into a rotation tensor $\mathbf{R}$ and into a deformation tensor $\mathbf{U}$

$$
\mathbf{F}_{m}^{n}=\mathbf{R}_{m}^{n} \mathbf{U}_{m}^{n} \text { with } \mathbf{U}_{m}^{n}=\mathbf{U}_{m}^{n \mathrm{~T}} \text { and } \mathbf{R}_{m}^{n \mathrm{~T}} \mathbf{R}_{m}^{n}=\mathbf{I} .
$$

Therefore one can define the Green-Lagrange deformation tensor $\mathbf{G L}_{m}^{n}$, the Almansi deformation tensor $\mathbf{A}_{m}^{n}$ and the natural deformation tensor $\mathbf{E}_{m}^{n}$ by

$$
\begin{aligned}
& \mathbf{G L}_{m}^{n} \equiv \frac{1}{2}\left[\mathbf{F}_{m}^{n \mathrm{~T}} \mathbf{F}_{m}^{n}-\mathbf{I}\right], \\
& \mathbf{A}_{m}^{n} \equiv \frac{1}{2}\left[\mathbf{I}-\mathbf{f}_{m}^{n} \mathbf{f}_{m}^{n}\right], \\
& \mathbf{E}_{m}^{n} \equiv \frac{1}{2} \ln \left[\mathbf{F}_{m}^{n}{ }_{m}^{n} \mathbf{F}_{m}^{n}\right] .
\end{aligned}
$$

Cauchy stress tensor at configuration $n$ is denoted $\boldsymbol{\Sigma}^{n}$. Let $\mathscr{H}_{i j k l}=k \delta_{i j} \delta_{k l}+G \delta_{i k} \delta_{j l}+G \delta_{i l} \delta_{j k}-$ $(2 G / 3) \delta_{i j} \delta_{k l}$ be the Hooke fourth-order tensor with operation $\mathscr{H}: \mathbf{E}$ defined by $\mathscr{H}_{i j k l} \mathbf{E}_{k l}$ and with $k$ the bulk modulus and $G$ the shear modulus. Let us denote the corotational values (i.e. before the rotation operation) with a superscript $c$. Then the corotational stress tensor is computed from

$$
\boldsymbol{\Sigma}^{c n+1}\left[\mathbf{\Sigma}^{n}+\mathscr{H}: \mathbf{E}_{n}^{n+1}-\mathbf{s}^{c}\right],
$$

where $\mathbf{s}^{c}$ is a deviatoric correcting term resulting from the $\mathbf{J} 2$ plasticity and computed from the radial return mapping [19]. It is computed in the following way. Let the elastic predictor $\mathbf{s}^{e}$ be the deviatoric part of $\boldsymbol{\Sigma}^{n}+\mathscr{H}: \mathbf{E}_{n}^{n+1}$. If the elastic predictor lies outside the von Mises criterion in the stress space, the step was, at least partially plastic and some corrections need to be introduced in the system. This correction is evaluated in the following way. The unit normal tensor $\mathbf{N}^{c}$ (normal to von Mises criterion in the stress space) is defined from $\mathbf{s}^{e} / \sqrt{\mathbf{s}^{e}: \mathbf{s}^{e}}$ where the operation $\mathbf{a}: \mathbf{b}$ is defined by $\mathbf{a}_{i j} \mathbf{b}_{i j}$. Let $\varepsilon^{p}$ be the equivalent plastic strain and let $\Sigma_{v}$ be the yield stress. Therefore, $\gamma^{p}$ is a scalar such that [19]

$$
\left[\varepsilon^{p}\right]^{n+1}=\left[\varepsilon^{p}\right]^{n}+\sqrt{\frac{2}{3}} \gamma^{p} \text { and } \mathbf{s}^{c}=2 G \gamma^{p} \mathbf{N}^{c}
$$

and obtained from the von Mises criterion

$$
\left[\mathbf{s}^{e}-2 G \gamma^{p} \mathbf{N}^{c}\right]:\left[\mathbf{s}^{e}-2 G \gamma^{p} \mathbf{N}^{c}\right]=\frac{2}{3}\left[\sum_{v}^{n+1}\left(\gamma^{p}\right)\right]^{2} .
$$

The final rotation scheme leads to

$$
\boldsymbol{\Sigma}^{n+1}=\mathbf{R}_{n}^{n+1} \boldsymbol{\Sigma}^{c n+1} \mathbf{R}_{n}^{n+1}{ }^{\mathrm{T}} .
$$

Let us note that such a formalism can be obtained with an heredity tensor defining the kinematic hardening [9].

\subsection{Expression of the internal forces}

The internal forces at time $t^{n}$ are expressed by Eq. (15). In [9], we have proposed the following expression of the internal forces for the EMCA integration scheme

$$
\left[\vec{F}_{\text {int }}^{n+1 / 2}\right]^{\xi}=\frac{1}{2}\left[\vec{F}_{\text {int }}^{*}+\vec{F}_{\text {int }}^{* *}\right]^{\xi},
$$




$$
\begin{aligned}
& {\left[\vec{F}_{\text {int }}^{*}\right]^{\xi}=\frac{1}{2} \int_{\mathbb{V}_{0}}\left\{\left[\mathbf{I}+\mathbf{F}_{n}^{n+1}\right]\left[\mathbf{\Sigma}^{n \mathrm{~T}}+\mathbf{C}^{*} \mathbf{f}_{0}^{n \mathrm{~T}} \vec{D}^{\xi} J_{0}^{n}\right\} \mathrm{d} \mathbb{V}_{0},\right.} \\
& {\left[\vec{F}_{\text {int }}^{* *}\right]^{\xi}=\frac{1}{2} \int_{\mathbb{V}_{0}}\left\{\left[\mathbf{I}+\mathbf{f}_{n}^{n+1}\right]\left[\boldsymbol{\Sigma}^{n+1} \mathrm{~T}+\mathbf{C}^{* *} \mathbf{f}_{0}^{n+1} \vec{D}^{\xi} J_{0}^{n+1}\right\} \mathrm{d} \mathbb{V}_{0},\right.}
\end{aligned}
$$

where $\mathbf{C}^{*}$ and $\mathbf{C}^{* *}$ are two correcting tensors resulting from the plasticity.

\subsubsection{Conservation of linear momentum}

Conservation of the linear momentum discretized in Eq. (26) is directly obtained from Eq. (41) since $\sum_{\xi} \vec{D}^{\xi}=0$.

\subsubsection{Conservation of angular momentum}

Conservation of the angular momentum discretized in Eq. (28) results from the symmetric nature of $\boldsymbol{\Sigma}, \mathbf{C}^{*}$ and $\mathbf{C}^{* *}$. Effectively, after some algebra [9], and if $\varepsilon$ is the third-order permutation tensor such that $\vec{a} \wedge \vec{b}=\varepsilon:[\vec{a} \otimes \vec{b}]$, with operation $[\vec{a} \otimes \vec{b}]_{i j}=\vec{a}_{i} \vec{b}_{j}$, Eq. (41) yields

$$
\begin{aligned}
{\left[\vec{x}^{n+1 / 2}\right]^{\xi} \wedge\left[\vec{F}_{\text {int }}^{*}\right]^{\xi} } & =\frac{1}{4} \varepsilon: \int_{\mathbb{V}_{0}}\left\{\left[\mathbf{I}+\mathbf{F}_{n}^{n+1}\right]\left[\mathbf{\Sigma}^{n}+\mathbf{C}^{*}\right]\left[\mathbf{I}+\mathbf{F}_{n}^{n+1}\right]^{\mathrm{T}} J_{0}^{n}\right\} \mathrm{d} \mathbb{V}_{0} \\
& =\int_{\mathbb{V}_{0}}\left\{\varepsilon: \Theta J_{0}^{n}\right\} \mathrm{d} \mathbb{V}_{0}
\end{aligned}
$$

that is equivalent to zero since $\boldsymbol{\Theta}=\left[\mathbf{I}+\mathbf{F}_{n}^{n+1}\right]\left[\mathbf{\Sigma}^{n}+\mathbf{C}^{*}\right]\left[\mathbf{I}+\mathbf{F}_{n}^{n+1}\right]^{\mathrm{T}}$ is a symmetric tensor and $\varepsilon$ is an antisymmetric tensor. The same technique leads to $\left[\vec{x}^{n+1 / 2}\right]^{\xi} \wedge\left[\vec{F}_{\text {int }}^{* *}\right]^{\xi}=0$.

\subsubsection{Conservation of energy}

The first thermodynamics principle discretized by (32) cannot be directly obtained for an hypoelastic model since no internal potential can be defined. Some algebra [9] yields

$$
\begin{aligned}
& {\left[\vec{F}_{\mathrm{int}}^{n+1 / 2}\right]^{\xi} \cdot\left[\vec{x}^{n+1}-\vec{x}^{n}\right]^{\xi}} \\
& \quad=\frac{1}{2} \int_{\mathbb{V}_{0}}\left\{\mathbf{G L}_{n}^{n+1}:\left[\mathbf{\Sigma}^{n}+\mathbf{C}^{*}\right] J_{0}^{n}+\mathbf{A}_{n}^{n+1}:\left[\mathbf{\Sigma}^{n+1}+\mathbf{C}^{* *}\right] J_{0}^{n+1}\right\} \mathrm{d} \mathbb{V}_{0}
\end{aligned}
$$

that has to be equal to $U_{\text {int }}^{n+1}-U_{\text {int }}^{n}+\Delta_{\text {int }}$ to verify Eq. (32). In [9], we have proposed to study Eq. (43) on a two-step cycle. The first step is an elasto-plastic loading step resulting in an internal dissipation $\Delta_{\text {int }}$, and the second step is an elastic unloading. Let $\mathbf{E}^{\text {el }}$ be the elastic part of the natural deformations and $\mathbf{U}_{n}^{\mathrm{e}}{ }^{n+1}$ be the elastic part of the deformation

$$
\mathscr{H}: \mathbf{E}_{n}^{\mathrm{e}^{n+1}} \equiv \mathscr{H}: \mathbf{E}_{n}^{n+1}-\mathbf{s}^{c} \text { and } \mathbf{E}_{n}^{\mathrm{e}^{n+1}} \equiv \frac{1}{2} \ln \left[\mathbf{U}_{n}^{\mathrm{e}^{n+1}} \mathbf{U}_{n}^{\mathrm{e}^{n+1}}\right] .
$$

The existence of $\mathbf{U}_{n}^{\mathrm{e} n}{ }^{n+1}$ is ensured from the symmetric nature of $\mathbf{E}_{n}^{\mathrm{e}^{n+1}}$. Therefore elastic tensor $\mathbf{G L}_{n}^{\mathrm{e}^{n+1}}$ and $\mathbf{A}_{n}^{\mathrm{e}^{n+1}}$ are defined from $\mathbf{U}_{n}^{\mathrm{e}^{n+1}}$

$$
\begin{aligned}
& \mathbf{G L}_{n}^{\mathrm{e}^{n+1}} \equiv \frac{1}{2}\left[\mathbf{U}_{n}^{\mathrm{e} \mathrm{e}^{n+1}} \mathbf{U}_{n}^{\mathrm{e}^{n+1}}-\mathbf{I}\right], \\
& \mathbf{A}_{n}^{\mathrm{e}^{n+1}} \equiv \frac{1}{2} \mathbf{R}_{n}^{n+1}\left[\mathbf{I}-\mathbf{U}_{n}^{\mathrm{e}^{n+1}} \mathbf{U}_{n}^{\mathrm{e}^{n+1}-1}\right] \mathbf{R}_{n}^{n+1^{\mathrm{T}}} .
\end{aligned}
$$


Elastic parts $\mathbf{G L}^{\mathrm{el}}$ and $\mathbf{A}^{\mathrm{el}}$ of, respectively, $\mathbf{G L}$ and $\mathbf{A}$, contribute to an increase of the reversible energy (i.e. energy that is stored as internal energy and could be released by the material [9]). Plastic part $\mathbf{G L}^{\mathrm{pl}}$ and $\mathbf{A}^{\mathrm{pl}}$ of, respectively, $\mathbf{G L}$ and $\mathbf{A}$ are obtained from

$$
\mathbf{G L}_{n}^{\mathbf{p}^{n+1}} \equiv \mathbf{G L}_{n}^{n+1}-\mathbf{G L}_{n}^{\mathrm{e}^{n+1}} \text { and } \mathbf{A}_{n}^{\mathrm{p}^{n+1}} \equiv \mathbf{A}_{n}^{n+1}-\mathbf{A}_{n}^{\mathrm{e}^{n+1}} \text {. }
$$

We have proved in [9] that Eq. (43), studied on the loading unloading cycle yields to

$$
\begin{aligned}
\Delta_{\text {int }}= & \frac{1}{2} \int_{V^{0}}\left\{\mathbf{G L}_{n} \mathbf{L}^{\mathrm{p}^{n+1}}: \mathbf{\Sigma}^{n} J_{0}^{n}\right\} \mathrm{d} V_{0}+\frac{1}{2} \int_{V^{0}}\left\{\mathbf{G} \mathbf{L}_{n}^{n+1}: \mathbf{C}^{*} J_{0}^{n}\right\} \mathrm{d} V_{0} \\
& +\frac{1}{2} \int_{V^{0}}\left\{\mathbf{A}_{n}^{\mathrm{p}^{1}{ }^{n+1}}: \boldsymbol{\Sigma}^{n+1} J_{0}^{n+1}\right\} \mathrm{d} V_{0}+\frac{1}{2} \int_{V^{0}}\left\{\mathbf{A}_{n}^{n+1}: \mathbf{C}^{* *} J_{0}^{n+1}\right\} \mathrm{d} V_{0} .
\end{aligned}
$$

To be physically consistent, Eq. (47) has to be related with a physical quantity. The positive internal plastic dissipation can be expressed from a volumic dissipation $D_{\text {int }}$ obtained from [10]

$$
D_{\text {int }} \simeq \frac{1}{2} \varepsilon_{n}^{p n+1} \sum_{v}^{n+1} J_{0}^{n+1}+\frac{1}{2} \varepsilon_{n}^{p n+1} \sum_{v}^{n} J_{0}^{n} .
$$

Let us define the tensors $\mathbf{C}^{*}$ and $\mathbf{C}^{* *}$ such that [10]

$$
\begin{aligned}
& \mathbf{C}^{*}=\frac{\left(D_{\text {int }} / J_{0}^{n}\right)-\mathbf{\Sigma}^{n}: \mathbf{G L}_{n}^{\mathrm{p} 1^{n+1}}}{\mathbf{G L}_{n}^{n+1}: \mathbf{G L}_{n}^{n+1}} \mathbf{G L}_{n}^{n+1}, \\
& \mathbf{C}^{* *}=\frac{\left(D_{\text {int }} / J_{0}^{n+1}\right)-\mathbf{\Sigma}^{n+1}: \mathbf{A}_{n}^{p l^{n+1}}}{\mathbf{A}_{n}^{n+1}: \mathbf{A}_{n}^{n+1}} \mathbf{A}_{n}^{n+1} .
\end{aligned}
$$

These tensors are symmetric (leading to the conservation of the angular momentum) and are equal to zero when no plastic deformation occurs, as assumed. Therefore Eq. (47) can be rewritten

$$
\Delta_{\text {int }}=\int_{V_{0}}\left\{D_{\text {int }}\right\} \mathrm{d} V_{0} \geqslant 0
$$

that is consistent with the first thermodynamics principle.

\subsection{Expression of the dissipation forces}

In the previous section, it was shown that tensors $\mathbf{C}^{*}$ and $\mathbf{C}^{* *}$ defined in Eq. (49) and used in Eq. (41), control the internal dissipation through Eq. (47). Therefore, we can control the numerical dissipation in the following way. Let $D_{W}$ be a volumic potential. We propose the new formulation of the dissipation forces

$$
\begin{aligned}
& {\left[\vec{F}_{\text {diss }}^{n+1 / 2}\right]^{\xi}=\frac{1}{2}\left[\vec{F}_{\text {diss }}^{*}+\vec{F}_{\text {diss }}^{* *}\right]^{\xi},} \\
& {\left[\vec{F}_{\text {diss }}^{*}\right]^{\xi}=\frac{1}{2} \int_{\mathbb{V}_{0}}\left\{\left[\mathbf{I}+\mathbf{F}_{n}^{n+1}\right] \mathbf{D}^{*} \mathbf{f}_{0}^{n \mathrm{~T}} \vec{D}^{\xi} J_{0}^{n}\right\} \mathrm{d} \mathbb{V}_{0},} \\
& {\left[\vec{F}_{\text {dis }}^{* *}\right]^{\xi}=\frac{1}{2} \int_{\mathbb{V}_{0}}\left\{\left[\mathbf{I}+\mathbf{f}_{n}^{n+1}\right] \mathbf{D}^{* *} \mathbf{f}_{0}^{n+1} \vec{D}^{\xi} J_{0}^{n+1}\right\} \mathrm{d} \mathbb{V}_{0}}
\end{aligned}
$$


with

$$
\mathbf{D}^{*}=\frac{\left(D_{W} / J_{0}^{n}\right)}{\mathbf{G L}_{n}^{n+1}: \mathbf{G L}_{n}^{n+1}} \mathbf{G L}_{n}^{n+1} \quad \text { and } \quad \mathbf{D}^{* *}=\frac{\left(D_{W} / J_{0}^{n+1}\right)}{\mathbf{A}_{n}^{n+1}: \mathbf{A}_{n}^{n+1}} \mathbf{A}_{n}^{n+1} .
$$

The potential $D_{W}$ must verify the following conditions:

$$
\begin{aligned}
& \frac{D_{W}}{\sqrt{\mathbf{G L}_{n}^{n+1}: \mathbf{G L}_{n}^{n+1}}} \rightarrow 0 \text { if } \mathbf{U}_{n}^{n+1} \rightarrow \mathbf{I}, \\
& \frac{D_{W}}{\sqrt{\mathbf{A}_{n}^{n+1}: \mathbf{A}_{n}^{n+1}}} \rightarrow 0 \text { if } \mathbf{U}_{n}^{n+1} \rightarrow \mathbf{I} .
\end{aligned}
$$

The algorithm will be second-order accurate if $\vec{F}_{\text {diss }}=\mathcal{O}\left(\Delta t^{2}\right)$. Therefore, the algorithm is secondorder accurate if $D_{W}=\mathcal{O}\left(\Delta t^{3}\right)$ and is first-order accurate if $D_{W}=\mathcal{O}\left(\Delta t^{2}\right)$. We propose the following expression of $D_{W}$, that satisfies Eq. (53), and that leads to a first-order accurate scheme.

$$
D_{W}=\frac{\chi}{2} \mathbf{E}_{n}^{\mathrm{el}^{n+1}}: \mathscr{H}: \mathbf{E}_{n}^{\mathrm{e}^{n+1}} J_{0}^{n} \geqslant 0,
$$

where $\chi$ is a user-defined parameter that controls the numerical dissipation. Its variation range will be studied in a future section. A second-order accurate algorithm could be reached if Eq. (54) is substituted by $D_{W}=\frac{\chi}{2} \mathbf{E}_{n}^{* n+1}: \mathscr{H}: \mathbf{E}_{n}^{\mathrm{e}^{n+1}} J_{0}^{n}$ where $\mathbf{E}^{*}$ is computed from an intermediate configuration $\vec{x}^{*}=\vec{x}^{n}+\mathcal{O}\left(\Delta t^{2}\right)$. Nevertheless, such an algorithm leads to an increase of the number of equations to be solved and is therefore more expensive [12]. In this paper we focused on first-order accurate schemes.

\subsubsection{Conservation of linear momentum}

Conservation of the linear momentum discretized in Eq. (26) is directly obtained from Eq. (51) since $\sum_{\xi} \vec{D}^{\xi}=0$.

\subsubsection{Conservation of angular momentum}

Conservation of the angular momentum discretized in Eq. (28) results from the symmetric nature of $\mathbf{D}^{*}$ and $\mathbf{D}^{* *}$. Proceeding such as with the internal forces, Eq. (51) yields

$$
\left[\vec{x}^{n+1 / 2}\right]^{\xi} \wedge\left[\vec{F}_{\text {diss }}^{*}\right]^{\xi}=\frac{1}{4} \int_{\mathbb{V}_{0}}\left\{\varepsilon:\left[\mathbf{I}+\mathbf{F}_{n}^{n+1}\right] \mathbf{D}^{*}\left[\mathbf{I}+\mathbf{F}_{n}^{n+1}\right]^{\mathrm{T}} J_{0}^{n}\right\} \mathrm{d} \mathbb{V}_{0},
$$

that is equivalent to zero since $\left[\mathbf{I}+\mathbf{F}_{n}^{n+1}\right] \mathbf{D}^{*}\left[\mathbf{I}+\mathbf{F}_{n}^{n+1}\right]^{\mathrm{T}}$ is a symmetric tensor and $\varepsilon$ is an antisymmetric tensor. The same technique leads to $\left[\vec{x}^{n+1 / 2}\right]^{\xi} \wedge\left[\vec{F}_{\text {diss }}^{* *}\right]^{\xi}=0$.

\subsubsection{Evaluation of the numerical dissipation}

The forces part of the numerical dissipation $\left(\Delta_{W}\right)$ expressed in Eq. (33) is obtained directly from Eq. (51)

$$
\begin{aligned}
& \Delta_{W}=\left[\vec{F}_{\text {diss }}^{n+1 / 2}\right]^{\xi} \cdot\left[\vec{x}^{n+1}-\vec{x}^{n}\right]^{\xi} \\
& \quad=\frac{1}{2} \int_{\mathbb{V}_{0}}\left\{\mathbf{G L}_{n}^{n+1}: \mathbf{D}^{*} J_{0}^{n}+\mathbf{A}_{n}^{n+1}: \mathbf{D}^{* *} J_{0}^{n+1}\right\} \mathrm{d} \mathbb{V}_{0}=\int_{\mathbb{V}_{0}}\left\{D_{W}\right\} \mathrm{d} \mathbb{V}_{0},
\end{aligned}
$$


that is always positive. The next goal to achieve is to determine the expression of the dissipation velocities.

\subsection{Expression of the dissipation velocities}

The dissipation velocities have to verify Eq. (29) and the velocity part of Eq. (33). Moreover, the expression of the dissipation velocity must lead to a spectral analysis without bifurcation of the spectral eigenvalues to achieve the numerical dissipation of the high frequencies. Armero and Romero [11,12] propose the following expression of the dissipation forces:

$$
M^{\xi \mu}\left[\vec{G}_{\mathrm{diss}}^{n+1 / 2}\right]^{\mu}=\int_{\mathbb{V}_{0}}\left\{\rho_{0} \varphi^{\xi} D_{K} \frac{\dot{\vec{x}}^{n+1}+\dot{\vec{x}}^{n}}{\left\|\dot{\vec{x}}^{n+1}\right\|^{2}-\left\|\dot{\vec{x}}^{n}\right\|^{2}}\right\} \mathrm{d} \mathbb{V}_{0}
$$

with $\dot{\vec{x}}=\varphi^{\xi} \dot{\vec{x}}^{\xi}$ and $\|\dot{\vec{x}}\|=\varphi^{\xi}\left\|\dot{\vec{x}}^{\xi}\right\|$ and with the specific kinetic potential $D_{K}$ that must verify

$$
\frac{D_{K}^{\xi}}{\left\|\left[\dot{\vec{x}}^{n+1}\right]^{\xi}\right\|-\left\|\left[\dot{\vec{x}}^{n}\right]^{\xi}\right\|} \rightarrow 0 \text { if }\left\|\left[\dot{\vec{x}}^{n+1}\right]^{\xi}\right\|-\left\|\left[\dot{\vec{x}}^{n}\right]^{\xi}\right\| \rightarrow 0 .
$$

Since the dissipation velocities are first-order accurate, the dissipation forces are also chosen firstorder accurate. Therefore we want $D_{K}=\mathcal{O}\left(\Delta t^{2}\right)$ such as

$$
D_{K}=\chi \frac{1}{2}\left[\left\|\dot{\vec{x}}^{n+1}\right\|-\left\|\dot{\vec{x}}^{n}\right\|\right]^{2} \geqslant 0
$$

This expression differs from the one proposed by Armero and Romero (i.e. $D_{K}=\chi\left\|\dot{\vec{x}}^{n+1}-\dot{\vec{x}}^{n}\right\|^{2}$ ) that does not lead to zero for an uniform rigid body rotation. A lumped expression of Eq. (57) can also be used to improve the performance of the algorithm. It leads (without sum on superscript $\xi$, but with velocities evaluated at node $\xi$ )

$$
\left[\vec{G}_{\text {diss }}^{n+1 / 2}\right]^{\xi}=\frac{D_{K}^{\xi}}{\left\|\left[\dot{\vec{x}}^{n+1}\right]^{\xi}\right\|^{2}-\left\|\left[\dot{\vec{x}}^{n}\right]^{\xi}\right\|^{2}} \frac{\left[\dot{\vec{x}}^{n+1}+\dot{\vec{x}}^{n}\right]^{\xi}}{2} .
$$

Therefore, one gets (with the lumped mass matrix $m$ such that $M^{\xi \mu}=m^{\xi} \delta_{\xi \mu}$ )

$$
\sum_{\mu} M^{\xi} \mu\left[\vec{G}_{\text {diss }}^{n+1 / 2}\right]^{\mu} \simeq \delta_{\xi \mu} \sum_{\mu} \frac{m^{\mu} D_{K}^{\mu}}{\left\|\left[\dot{\vec{x}}^{n+1}\right]^{\mu}\right\|^{2}-\left\|\left[\dot{\vec{x}}^{n}\right]^{\mu}\right\|^{2}} \frac{\left[\dot{\vec{x}}^{n+1}+\dot{\vec{x}}^{n}\right]^{\mu}}{2} .
$$

Actually, combination of Eqs. (59) and (61) leads to the expression of the dissipation velocity

$$
\left[\vec{G}_{\mathrm{diss}}^{n+1 / 2}\right]^{\xi}=\chi \frac{\left\|\left[\dot{\vec{x}}^{n+1}\right]^{\xi}\right\|-\left\|\left[\dot{\vec{x}}^{n}\right]^{\xi}\right\|}{\left\|\left[\dot{\vec{x}}^{n+1}\right]^{\xi}\right\|+\left\|\left[\dot{\vec{x}}^{n}\right]^{\xi}\right\|} \frac{\left[\dot{\vec{x}}^{n+1}+\dot{\vec{x}}^{n}\right]^{\xi}}{2} .
$$




\subsubsection{Conservation of angular momentum}

Conservation of angular momentum Eq. (29) is verified since

$$
\begin{aligned}
& \sum_{\mu} \sum_{\xi}\left[\dot{\vec{x}}^{n+1}+\dot{\vec{x}}^{n}\right]^{\xi} \wedge M^{\xi}\left[\vec{G}_{\mathrm{diss}}^{n+1 / 2}\right]^{\mu} \\
& \quad=\sum_{\mu} \frac{m^{\mu} D_{K}^{\mu}}{\left\|\left[\dot{\vec{x}}^{n+1}\right]^{\mu}\right\|^{2}-\left\|\left[\dot{\vec{x}}^{n}\right]^{\mu}\right\|^{2}} \frac{\left[\dot{\vec{x}}^{n+1}+\dot{\vec{x}}^{n}\right]^{\mu}}{2} \wedge\left[\dot{\vec{x}}^{n+1}+\dot{\vec{x}}^{n}\right]^{\mu}=0 .
\end{aligned}
$$

\subsubsection{Evaluation of the numerical dissipation}

The velocity part of numerical dissipation $\left(\Delta_{K}\right)$ defined by Eq. (33) is evaluated by (using Einstein's notations)

$$
\Delta_{K}=\left[\dot{\vec{x}}^{n+1}-\dot{\vec{x}}^{n}\right]^{\xi} \cdot M^{\xi \mu}\left[\vec{G}_{\text {diss }}^{n+1 / 2}\right]^{\mu}=m^{\mu} D_{K}^{\mu} \simeq \int_{\mathbb{V}_{0}}\left\{\rho_{0} D_{K}\right\} \mathrm{d} \mathbb{V}_{0} \geqslant 0 .
$$

\subsection{Spectral analysis}

At this point, we have proved that our formulation is physically consistent and numerically dissipative in the non-linear range. Now, we have to achieve the spectral analysis to prove that the high frequencies are numerically dissipated. The spectral analysis leads to properties of linear oscillators. So we restrict this demonstration to linearity. We expect these properties to be still verified in the non-linear range. Let us assume a small transformation, without plasticity, hypothesis with $\varepsilon_{i j}=\frac{1}{2}\left(\partial \vec{x}_{i} / \partial \vec{x}_{j}+\partial \vec{u}_{j} / \partial \vec{x}_{i}\right)$ the small strain tensor. Therefore one gets

$$
\begin{aligned}
& J_{0}^{n+1} \simeq J_{0}^{n} \text { and } \quad \mathbf{R}_{0}^{n} \simeq \mathbf{I} \\
& \boldsymbol{\varepsilon}_{n}^{\mathrm{el}^{n+1}} \simeq \mathbf{G L}_{n}^{\mathrm{el}^{n+1}} \simeq \mathbf{A}_{n}^{\mathrm{el}^{n+1}} \simeq \mathbf{E}_{n}^{\mathrm{e}^{n+1}} \\
& \mathbf{E}_{0}^{\mathrm{e}^{n+1}} \simeq \mathbf{E}_{0}^{\mathrm{el}^{n}}+\mathbf{E}_{n}^{\mathrm{e}^{n+1}} \text { and } \boldsymbol{\Sigma}^{n} \simeq \mathscr{H}: \mathbf{E}_{0}^{\mathrm{e}^{n}} .
\end{aligned}
$$

Let us assume a uniform tension problem with a displacement $x$. The elements are decomposed with linear shape functions. Therefore, it comes, for one element of length $l$ and of cross-section $A$, the following incremental deformation tensor holds:

$$
\boldsymbol{\varepsilon}_{n}^{n+1}=\mathbf{F}_{n}^{n+1}-\mathbf{I}=\frac{x^{n+1}-x^{n}}{l}\left(\begin{array}{ccc}
1 & 0 & 0 \\
0 & \frac{2 G-3 k}{6 k+2 G} & 0 \\
0 & 0 & \frac{2 G-3 k}{6 k+2 G}
\end{array}\right)
$$

with $k$ the bulk modulus and $G$ the shear modulus. Only the first component of the stress tensors is different from zero and it comes

$$
\boldsymbol{\Sigma}_{11}^{n}=\frac{x^{n}}{l} \frac{9 k G}{3 k+G} \quad \text { and } \quad \boldsymbol{\Sigma}_{11}^{n+1}=\frac{x^{n+1}}{l} \frac{9 k G}{3 k+G} .
$$


The first components of the forces vectors (41) and (51) become (at the first-order in $x$ ) with $Y=(9 k G) /(3 k+G)$ the Young's modulus

$$
\left[\vec{F}_{\mathrm{int}}^{n+1 / 2}\right]_{1}=\frac{Y A}{l} \frac{x^{n+1}+x^{n}}{2} \text { and }\left[\vec{F}_{\mathrm{diss}}^{n+1 / 2}\right]_{1}=\frac{Y A}{l} \chi \frac{x^{n+1}-x^{n}}{2} .
$$

For a translation motion, the kinematic potential (59) is rewritten as $D_{\mathrm{K}}=\chi \frac{1}{2}\left[\dot{\vec{x}}^{n+1}-\dot{\vec{x}}^{n}\right]^{2}$ that is correct while the velocity keeps the same direction between two steps. Since the lumped mass $m$ associated for each extremity is equal to $\rho l A / 2$, if $\omega^{2}=18 k G /\left([3 k+G] \rho l^{2}\right)$, then Eqs. (17)-(19) lead to

$$
\begin{aligned}
& x^{n+1}=x^{n}+\frac{\Delta t}{2}\left[1+\chi \frac{\dot{x}^{n+1}-\dot{x}^{n}}{\dot{x}^{n+1}+\dot{x}^{n}}\right]\left[\dot{x}^{n+1}+\dot{x}^{n}\right], \\
& \dot{x}^{n+1}=\dot{x}^{n}+\frac{\Delta t}{2}\left[\ddot{x}^{n+1}+\ddot{x}^{n}\right], \\
& \ddot{x}^{n+1}=-\ddot{x}^{n}-\omega^{2}\left[1+\chi \frac{x^{n+1}-x^{n}}{x^{n+1}+x^{n}}\right]\left[x^{n+1}+x^{n}\right] .
\end{aligned}
$$

This system of equations is equivalent to the one obtained by Armero and Romero [11] for a spring model, and leads to the spectral matrix $\mathbf{A}^{\mathrm{s}}(\Omega)$

$$
\left(\begin{array}{c}
x^{n+1} \\
\Delta t \dot{x}^{n+1} \\
\Delta t^{2} \ddot{x}^{n+1}
\end{array}\right)=\underbrace{\left(\begin{array}{ccc}
\frac{1-\frac{\Omega^{2}}{4}\left[1-\chi^{2}\right]}{D(\Omega)} & \frac{1}{D(\Omega)} & 0 \\
\frac{-\Omega^{2}}{D(\Omega)} & \frac{1-\frac{\Omega^{2}}{4}\left[1-\chi^{2}\right]}{D(\Omega)} & 0 \\
\frac{-2 \Omega^{2}}{D(\Omega)} & \frac{-\Omega^{2}[1+\chi]}{D(\Omega)} & -1
\end{array}\right)}_{\mathbf{A}^{\mathrm{s}}(\Omega)}\left(\begin{array}{c}
x^{n} \\
\Delta t \dot{x}^{n} \\
\Delta t^{2} \ddot{x}^{n}
\end{array}\right)
$$

with $D(\Omega)=1+\Omega^{2} / 4[1+\chi]^{2}$ and with $\Omega=\omega \Delta t$. This system is reduced to a two-dimensional (2d) system with the two conjugate complex eigenvalues

$$
\lambda=\frac{1-\Omega^{2} / 4\left[1-\chi^{2}\right] \pm \mathrm{i} \Omega}{D(\Omega)} .
$$

Armero and Romero [11,12] pointed out the necessity of considering dissipation velocities unless there exists a bifurcation limit $\Omega_{\mathrm{b}}$ that leads to real eigenvalues. Let $\Omega_{\mathrm{d}}$ and $\xi_{\mathrm{d}}$, respectively, be the a-dimensional pulsation of the solution and the damp ratio of the solution

$$
\begin{aligned}
& \Omega_{\mathrm{d}}=\arctan \left(\frac{\mathfrak{J} \lambda_{1}}{\mathfrak{R} \lambda_{1}}\right) \simeq \Omega-\frac{1}{4}\left[\chi^{2}+\frac{1}{3}\right] \Omega^{3}+\mathcal{O}\left(\Omega^{4}\right), \\
& \xi_{\mathrm{d}}=\frac{-\ln \left(\left[\mathfrak{\Im} \lambda_{1}\right]^{2}+\left[\Re \lambda_{1}\right]^{2}\right)}{2 \Omega_{\mathrm{d}}} \simeq \frac{\chi}{2} \Omega+\mathcal{O}\left(\Omega^{2}\right) .
\end{aligned}
$$

It comes directly that the error on the pulsation $e_{\Omega}=\left(\Omega_{\mathrm{d}}-\Omega\right) / \Omega$ and that the error on the damping ratio $e_{\xi}=\xi_{\mathrm{d}}$ are both first-order in $\Delta t$, leading to a first-order accurate scheme. The 
spectral radius of the response is then evaluated by

$$
\rho_{\mathrm{d}}(\Omega)=\|\lambda\|=\frac{1}{1+\left(\Omega^{2} / 4\right)[1+\chi]^{2}} \sqrt{\left[1-\frac{\Omega^{2}}{4}\left[1-\chi^{2}\right]\right]^{2}+\Omega^{2}} .
$$

If $0 \leqslant \chi \leqslant 1$ the spectral radius is always lower than unity. It is close to unity for the low frequencies and tends to its minimal value $\rho_{\infty}=(1-\chi) /(1+\chi)$ for the high frequencies.

\section{Numerical examples}

In this section, we will analyze the results obtained with the developed formulation and with the traditional Newmark family on an academic case: the Taylor bar problem. Let us note that the final purpose of this paper is to demonstrate the accuracy and robustness of the implicit scheme. We have in mind combining the implicit scheme with an explicit one and therefore we need efficient time integration. We do not claim better results than an explicit method, but we also compared the solutions with a traditional central difference scheme. The following step will be to prove that our formulation is able to simulate a more complex problem such as a blade loss problem.

\subsection{Example 1: The Taylor bar}

The problem consists in a cylindrical bar (external diameter $d_{\mathrm{e}}=6.4 \mathrm{~mm}$, length $l=32.4 \mathrm{~mm}$ ) made of an elasto-plastic material (Young's modulus $Y=117 E 9 \mathrm{~N} / \mathrm{m}^{2}$, Poisson's ratio $v=0.35$, density $\rho=8930 \mathrm{~kg} / \mathrm{m}^{3}$, initial yield stress $\Sigma_{0}=400 \mathrm{~N} / \mathrm{mm}^{2}$, hardening parameter $\left.h=100 \mathrm{~N} / \mathrm{mm}^{2}\right)$ that impacts a rigid wall with an initial velocity $\left(\dot{x}_{0}=227 \mathrm{~m} / \mathrm{s}\right)$. We study one quarter of the cylinder. We use the discretization proposed by Meng and Laursen [8]. It is discretized into 12 times $48=576$ elements (Fig. 1). The simulation occurs in $80 \mu$ s. We will compare the results obtained with 4 algorithms. For each algorithm, the spectral radius for an infinite frequency is chosen as $\rho_{\infty}=0.7$. The different algorithms are

- the first-order accurate EDMC algorithm (EDMC-1) with $\chi=\left(1-\rho_{\infty}\right) /\left(1+\rho_{\infty}\right)$;

- the Newmark algorithm [1] with the first parameter $\beta=1 /\left(\left[1+\rho_{\infty}\right]^{2}\right)$ and the second parameter $\gamma=\left(3-\rho_{\infty}\right) /\left(2+2 \rho_{\infty}\right)$;

- the Hilber-Hughes-Taylor (HHT) [4] algorithm with the first Newmark parameter $\beta=1 /\left(\left[1+\rho_{\infty}\right]^{2}\right)$, the second Newmark parameter $\gamma=\left(3-\rho_{\infty}\right) /\left(2+2 \rho_{\infty}\right)$ and with the internal forces interpolation parameter $\alpha_{F}=\left(1-\rho_{\infty}\right) /\left(1+\rho_{\infty}\right)$;

- the Chung-Hulbert $(\mathrm{CH})$ [4] algorithm with the first Newmark parameter $\beta=1 /\left(\left[1+\rho_{\infty}\right]^{2}\right)$, the second Newmark parameter $\gamma=\left(3-\rho_{\infty}\right) /\left(2+2 \rho_{\infty}\right)$, with the internal forces interpolation

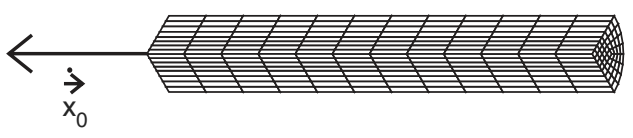

Fig. 1. Mesh of the Taylor bar. 
Table 1

Final results of the Taylor bar

\begin{tabular}{lllll}
\hline Scheme & $\Delta t(\mu \mathrm{s})$ & Radius & Length & Maximal $\varepsilon^{p}$ \\
\hline EDMC-1 & 1 & 0.006499 & 0.02211 & 2.43 \\
Newmark & 1 & 0.006628 & 0.02166 & 2.52 \\
HHT & 1 & 0.006636 & 0.02157 & 2.52 \\
CH & 1 & 0.006609 & 0.02152 & 2.47 \\
EDMC-1 & 0.5 & 0.006535 & 0.02189 & 2.43 \\
Newmark & 0.5 & 0.006628 & 0.02166 & 2.51 \\
HHT & 0.5 & 0.006601 & 0.02158 & 2.46 \\
CH & 0.5 & 0.006575 & 0.02154 & 2.44 \\
EDMC-1 & 0.1 & 0.006555 & 0.02166 & 2.40 \\
Newmark & 0.1 & 0.006593 & 0.02162 & 2.48 \\
HHT & 0.1 & 0.006555 & 0.02166 & 2.38 \\
CH & 0.1 & 0.006549 & 0.02156 & 2.38 \\
Central difference & $0.053-0.061$ & 0.006837 & 0.02163 & 2.54
\end{tabular}
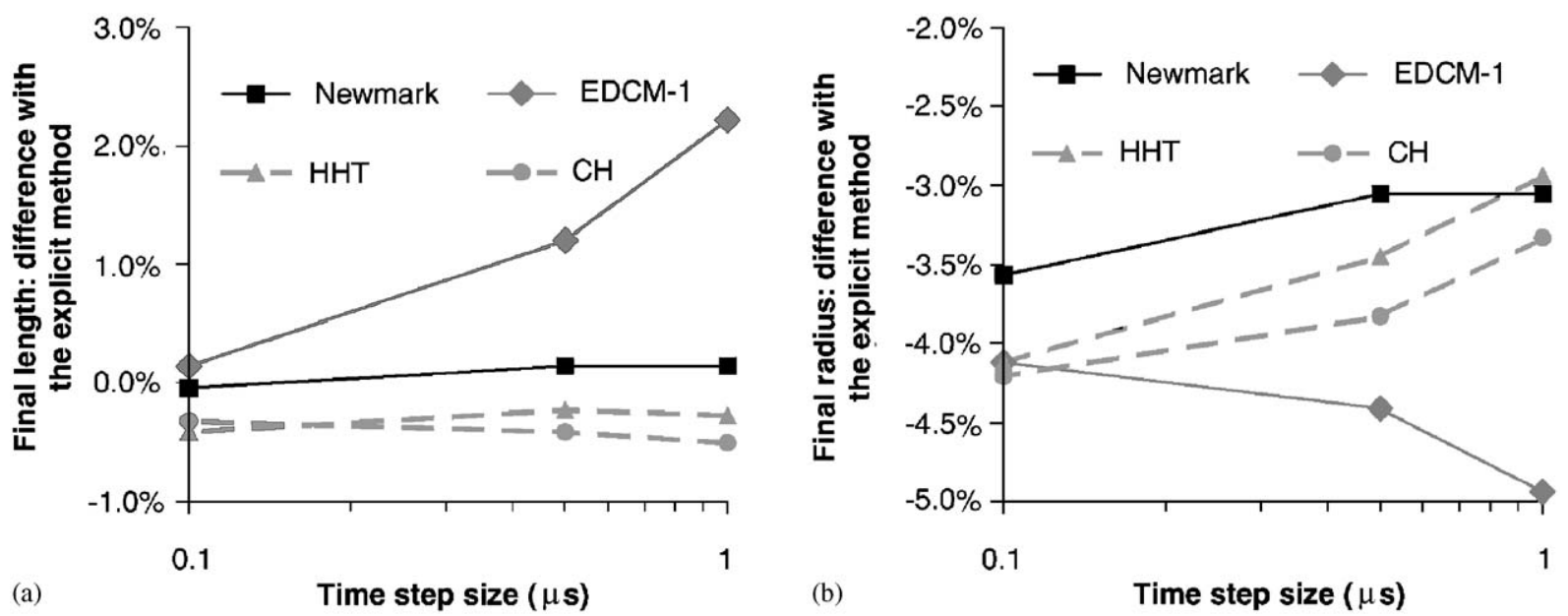

Fig. 2. Final results (after $80 \mu \mathrm{s})$ for the Taylor bar: difference with the explicit simulation.

parameter $\alpha_{F}=\rho_{\infty} /\left(1+\rho_{\infty}\right)$ and with the inertial forces interpolation parameter $\alpha_{M}=$ $\left(2 \rho_{\infty}-1\right) /\left(1+\rho_{\infty}\right)$;

- the explicit central difference scheme.

For each implicit algorithm we study the influence of the time step size that evolves in a range from $0.1-1 \mu \mathrm{s}$. For a time step size equal to $1.25 \mu \mathrm{s}$, only the Newmark and the EDMC-1 algorithms have converged. Final results are reported in Table 1 and compared to an explicit 

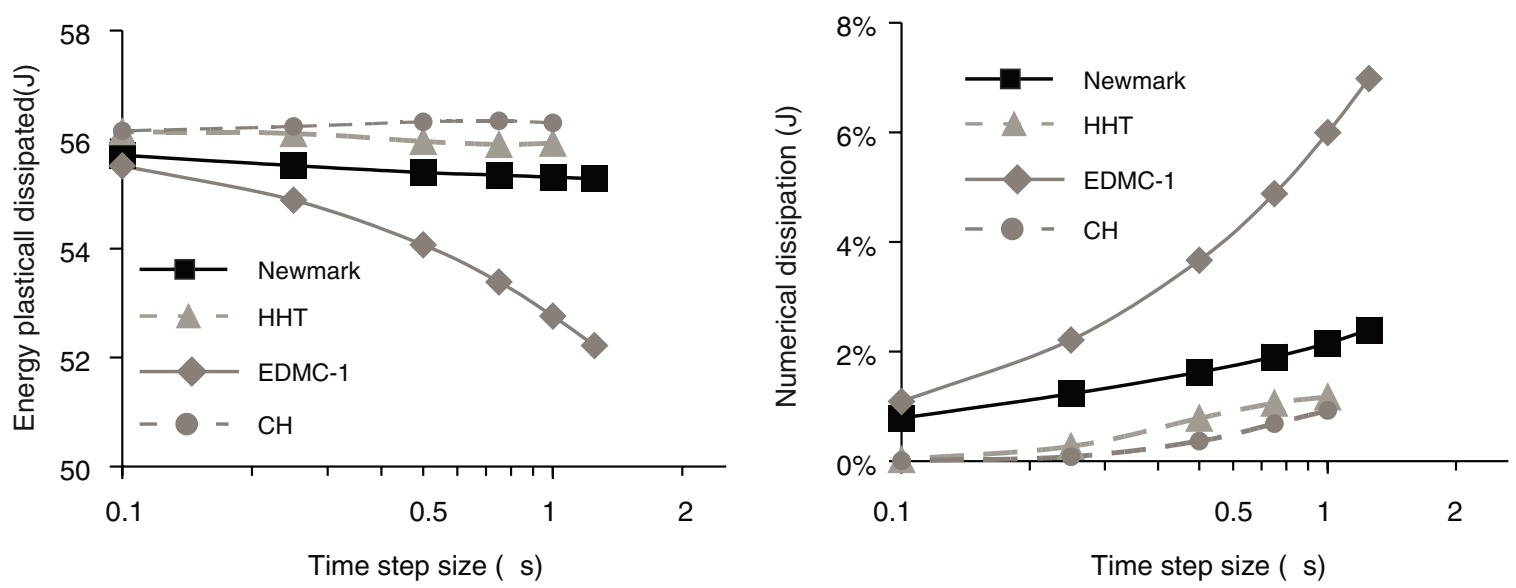

Fig. 3. Energy dissipated after $80 \mu$ s for the Taylor bar.
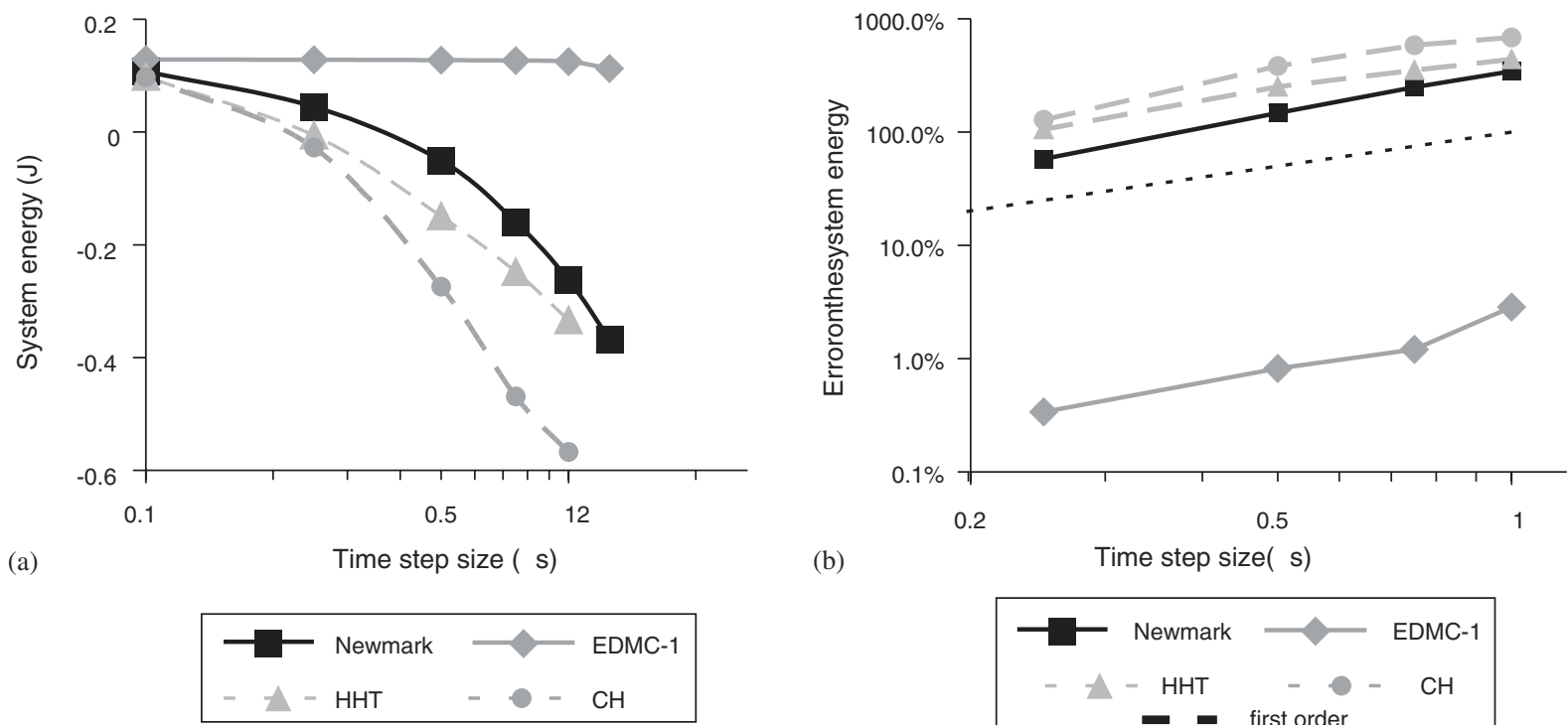

(b)

Time step size $(\mu s)$

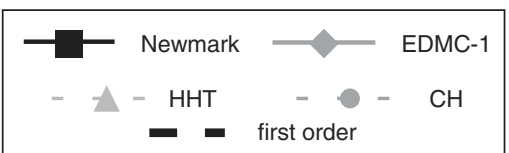

Fig. 4. Final energy of the Taylor bar after $80 \mu$ s: (a) energy, (b) error on the energy.

solution. If we compare the final length (Fig. 2a) it appears that the length difference with the explicit scheme increases with the time steps size and is positive for the EDMC-1 scheme. It can be easily understood since the energy numerically dissipated does not lead to deformation of the cylinder. For the other schemes the tendency is less clear. We have the same conclusions when analyzing the final radius (Fig. 2b). For the EDMC scheme, if the time step increases, the deformation of the beam decreases and the final radius is less important. This tendency is not respected for the other schemes. Finally, let us note that the differences are always lower than $5 \%$. That allows us to consider that since the deformation is directed due to the wave propagation, this last one is correctly integrated with the implicit scheme. This is confirmed when analysing the 
energy. Fig. 3a represents the evolution, with the time step size, of the physically (plastically) dissipated energy and Fig. 3(b) represents the energy numerically dissipated. In accordance with the theory, since there are no bifurcation in the spectral analysis, if the time step size is increased, the a-dimensional pulsation is increased and the energy numerically dissipated is increased. Surprisingly, if the numerical dissipation is increased, the $\mathrm{CH}$ scheme overestimates the plastically dissipated energy (Fig. 3a). For the other schemes, since a part of the initial kinetic energy is numerically dissipated, the plastically dissipated energy is underestimated. Fig. 4a illustrates the energy of the system (sum of the kinetic energy and the work of the internal forces minus the plastically dissipated energy) of the bar. We can see that only the EDMC-1 leads to positive energy for all the time step sizes, for the other schemes, if the time step increases, the plastically dissipated energy becomes larger than the work of the internal forces. Thus, these schemes violate the first principle of the thermodynamics. Fig. 4(b) illustrates the error on the system energy. All the scheme leads to first-order accurate solutions, while a linear theory predicts that the $\mathrm{CH}$ and of the HHT schemes are second-order accurate. We assume that in the non-linear range, the second-order accuracy is no longer ensured. Equivalent plastic strains of the final configurations are illustrated on Fig. 5. The solution obtained with a time step size equal to $1 \mu$ s and with the EDMC-1 algorithm is equivalent within less than $1 \%$ to the solution obtained with a time step size equal to $0.1 \mu \mathrm{s}$. For the other schemes, solutions with $\Delta=1 \mu \mathrm{s}$ overestimate the equivalent plastic strain of about $5 \%$.

From this example it clearly appears that the proposed scheme leads to positive numerical dissipation and to accurate time integration for all the time steps studied. Nevertheless, it also appears that the accuracy of the scheme depends on the size of the time step. Therefore when dealing with complex problems we need to estimate the time step size from an integration error. We use a technique that constantly adapts this size to the dynamics [20,21]. Then, we can claim that the algorithm is stable and that the time step is small enough to correctly take into account the dynamics.

\subsection{Example 2: Blade loss problem in an aero engine}

A turbo-engine is modelled with a shaft that has an imposed revolution motion on one extremity. At the other extremity, there is a disk with 24 blades. The shaft, the disk and the blades are part of the rotor, which is in rotation in a stator. The stator is composed of a casing and a bearing.

The rotor has a cyclic symmetry of 15 degrees (24 blades). Fig. 6a illustrates a 15-degree-sector. The blade is defined thanks to a ruled surface that has two splines for extremities. The two splines are described in Table 2. The blades are made of an alloy (density $\rho=3600 \mathrm{~kg} / \mathrm{m}^{3}$, Young's modulus $Y=88000 \mathrm{~N} / \mathrm{mm}^{2}$, Poisson's ratio $v=0.31$, initial yield stress $\Sigma_{0}=880 \mathrm{~N} / \mathrm{mm}^{2}$ and hardening parameter $h=26700 \mathrm{~N} / \mathrm{mm}^{2}$ ). The disk and the shaft are composed of another alloy (density $\rho=6300 \mathrm{~kg} / \mathrm{m}^{3}$, Young's modulus $Y=165000 \mathrm{~N} / \mathrm{mm}^{2}$, Poisson's ratio $v=0.31$, initial yield stress $\Sigma_{0}=800 \mathrm{~N} / \mathrm{mm}^{2}$, hardening parameter $h=271 \mathrm{~N} / \mathrm{mm}^{2}$ ). The blade is discretized with 99 elements: 11 in length (elements at the head of the blade are 50\% smaller than at the root), 9 elements in height and 1 element through the thickness. The disk has 2 elements through the thickness and 72 elements on the circumference. The shaft has 1 element on its thickness and 11 elements on its length ( 8 for the constant section shaft and 3 for the conical part). The shaft has 72 elements on its circumference. The elements are 8-node bricks with constant pressure. 


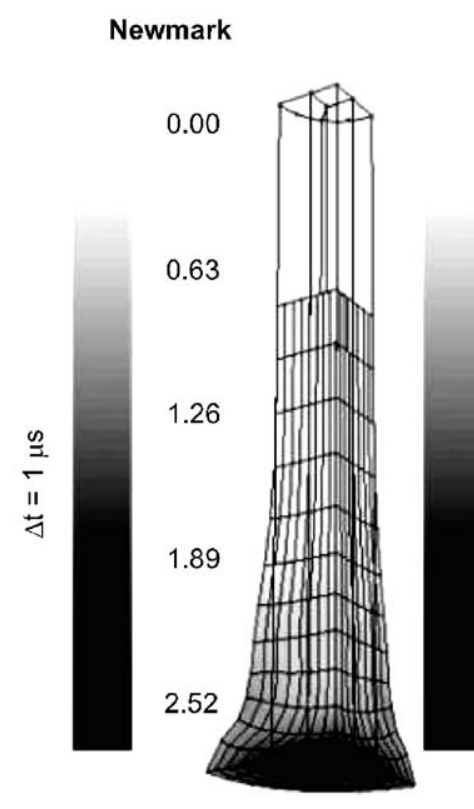

EDMC-1

HHT

$\mathrm{CH}$
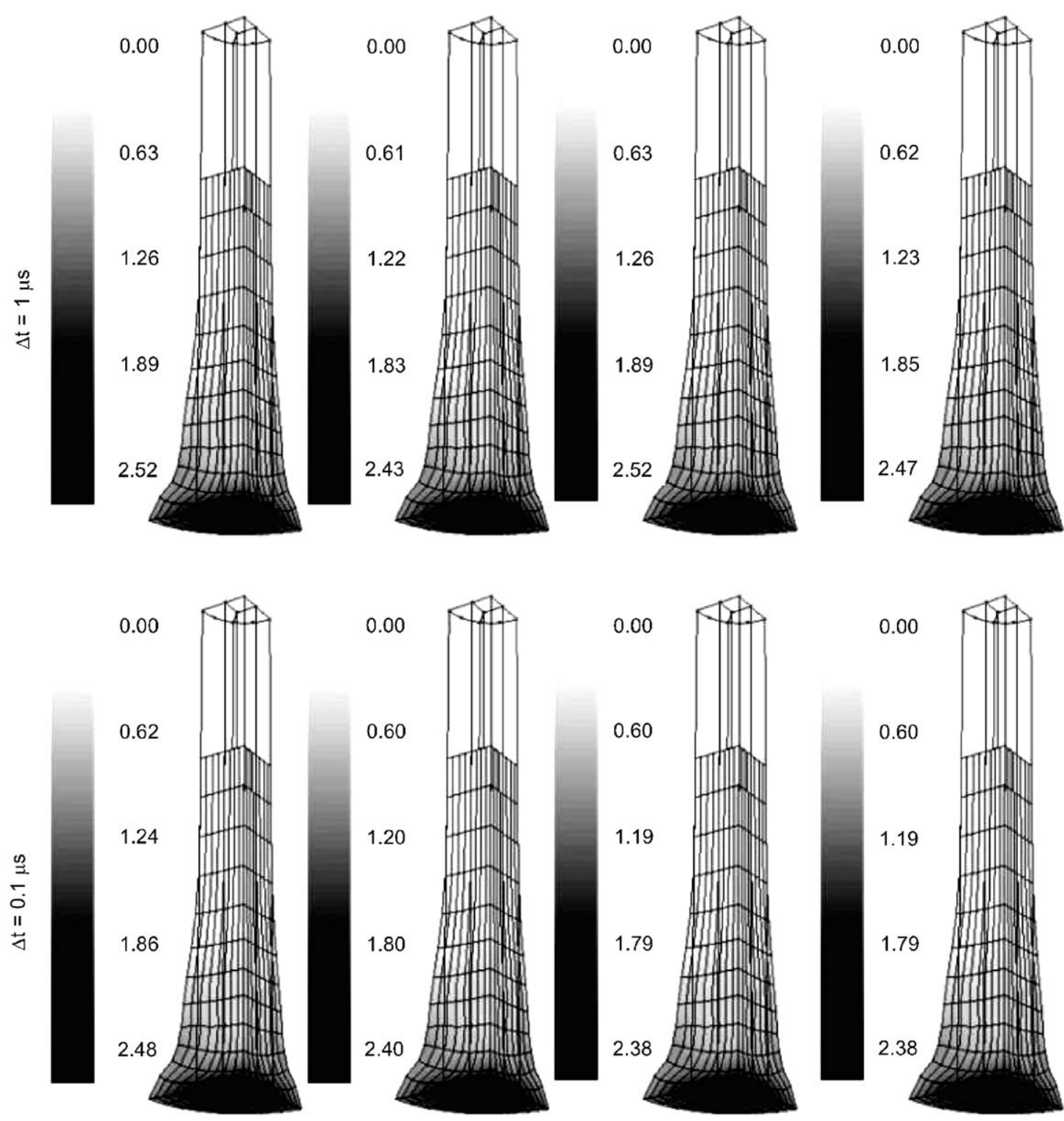

Fig. 5. Deformation and equivalent plastic strain (after $80 \mu \mathrm{s}$ ) for the Taylor bar.

At time $t=0 \mathrm{~s}$, the initial configuration of the rotor is equilibrated for a rotation velocity of $4775 \mathrm{rpm}$. This initial configuration is computed with a Newton-Raphson scheme where the external forces are the analytical inertial forces computed from the nodes position and from the imposed rotation velocity. The von Mises stresses resulting from this uniform rotation velocity are illustrated in Fig. 6b. The blade pointed by an arrow is independent from the disk. To evaluate the initial configuration, it is linked to the disk through an adhesion law (normal penalty $k_{\mathrm{p}}=10^{9}$, 

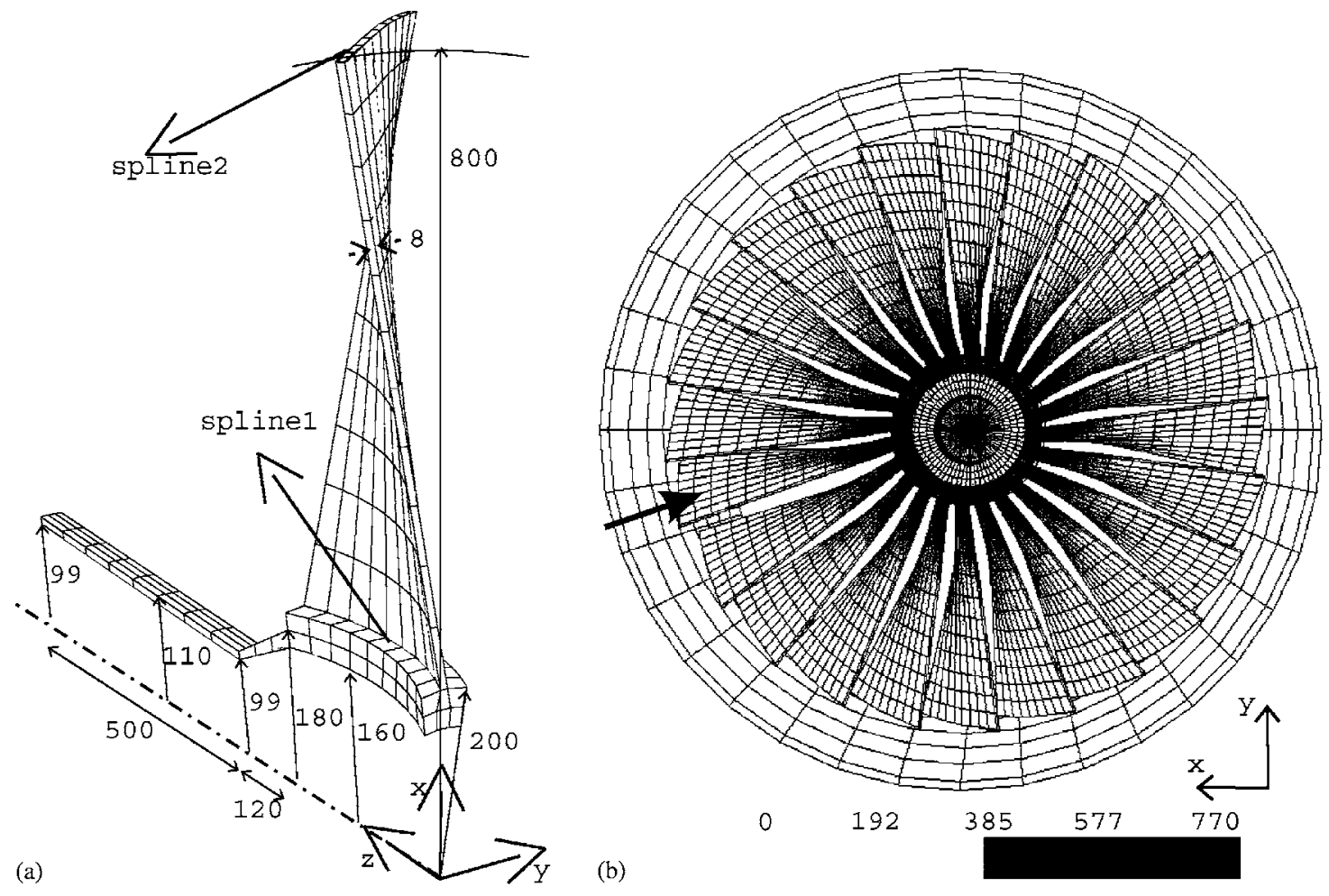

Fig. 6. Description of the problem model.

Table 2

Coordinates $(\mathrm{mm})$ of the splines

\begin{tabular}{lll}
\hline Point & Spline 1 & Spline 2 \\
\hline 1 & $\vec{x}=(200 ; 0 ; 0)$ & $\vec{x}=(791.9 ;-113.4 ; 8.9)$ \\
2 & $\vec{x}=(199.3 ; 8.9 ; 19.7)$ & $\vec{x}=(793.9 ;-94.7 ; 28.2)$ \\
3 & $\vec{x}=(198.8 ; 16.3 ; 39.9)$ & $\vec{x}=(795.1 ;-75.9 ; 47.5)$ \\
4 & $\vec{x}=(198.3 ; 22.1 ; 60.7)$ & $\vec{x}=(796.4 ;-57.0 ; 66.7)$ \\
5 & $\vec{x}=(198 ; 26.3 ; 81.9)$ & $\vec{x}=(797.6 ;-38.2 ; 85.9)$ \\
6 & $\vec{x}=(197.9 ; 28.9 ; 103.4)$ & $\vec{x}=(798.8 ;-19.4 ; 105.2)$ \\
7 & $\vec{x}=(197.8 ; 29.7 ; 125)$ & $\vec{x}=(800 ;-0.6 ; 124.4)$ \\
8 & $\vec{x}=(197.9 ; 28.9 ; 146.6)$ & $\vec{x}=(799.8 ; 18.3 ; 143.7)$ \\
9 & $\vec{x}=(198 ; 26.3 ; 168.0)$ & $\vec{x}=(799.1 ; 37.1 ; 162.9)$ \\
10 & $\vec{x}=(198.3 ; 22.1 ; 189.2)$ & $\vec{x}=(798.0 ; 55.7 ; 182.3)$ \\
11 & $\vec{x}=(198.8 ; 16.3 ; 210.1)$ & $\vec{x}=(796.5 ; 74.3 ; 201.8)$ \\
12 & $\vec{x}=(199.3 ; 8.9 ; 230.3)$ & $\vec{x}=(794.6 ; 92.6 ; 221.4)$ \\
13 & $\vec{x}=(200 ; 0 ; 0.25)$ & $\vec{x}=(792.3 ; 110.7 ; 241.3)$
\end{tabular}

tangential penalty $k_{\mathrm{T}}=10^{8}$ ). After the initial configuration is evaluated, in order to simulate the blade loss, this link is numerically removed thus creating some unbalance in the system and the free blade interacts with the other blades and with the casing. The interaction between the blades 

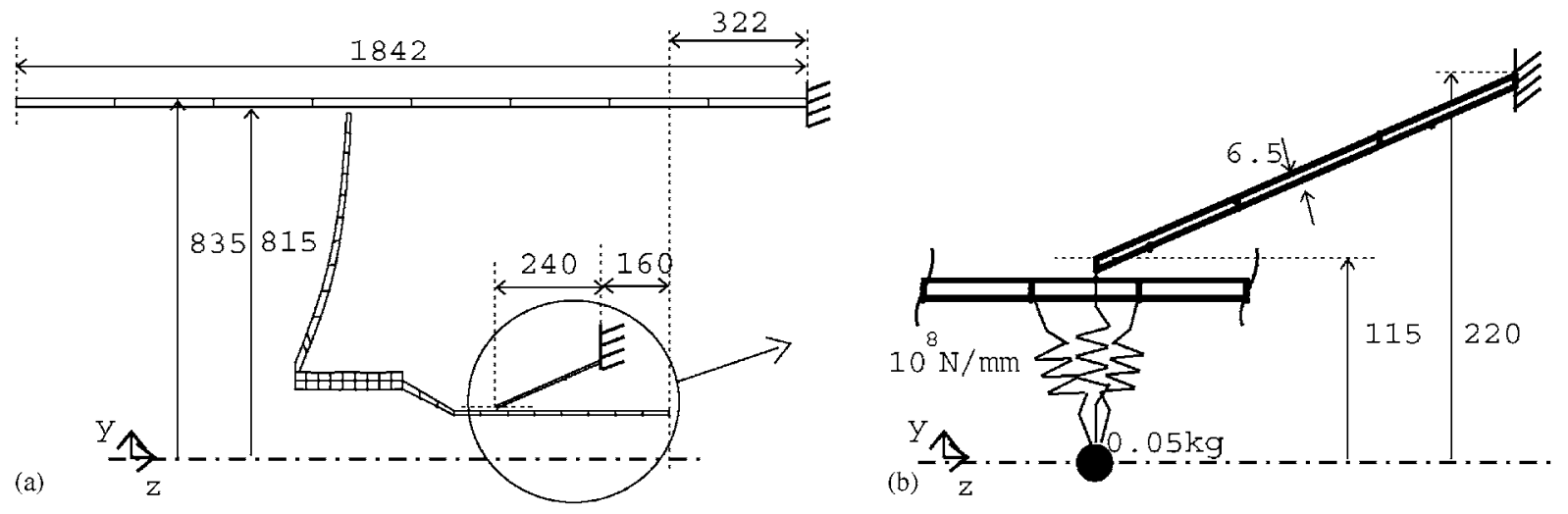

Fig. 7. Model of the stator (mm).

and the casing is simulated with a Coulomb friction law (normal penalty $k_{\mathrm{p}}=10^{9}$, tangential penalty $k_{\mathrm{T}}=10^{7}$, friction coefficient $\mu_{\mathrm{c}}=0.1$ ). The interaction between the free blade and the other blades is simulated with the same law. Contact interactions between attached blades are simulated with a frictionless law (normal penalty $k_{\mathrm{p}}=10^{9}$ ).

The casing is a cylinder made of an aluminum alloy (density $\rho=2710 \mathrm{~kg} / \mathrm{m}^{3}$, Young's modulus $Y=55200 \mathrm{~N} / \mathrm{mm}^{2}$, Poisson's ratio $v=0.31$, yield stress $\Sigma_{0}=550 \mathrm{~N} / \mathrm{mm}^{2}$ and hardening parameter $h=281 \mathrm{~N} / \mathrm{mm}^{2}$ ). Its geometry is illustrated in Fig. 7a. The bearing has a conical geometry (Fig. 7a and b) and is made of an alloy (density $\rho=3600 \mathrm{~kg} / \mathrm{m}^{3}$, Young's modulus $Y=88000 \mathrm{~N} / \mathrm{mm}^{2}$, Poisson's ratio $v=0.31$, yield stress $\Sigma_{0}=550 \mathrm{~N} / \mathrm{mm}^{2}$ and hardening parameter $h=2600 \mathrm{~N} / \mathrm{mm}^{2}$ ). The displacement of the shaft is restrained by the bearing thanks to a central node (Fig. 7b). There are springs between the central node and the extremity nodes of the bearing and there are springs between the central node and two rows on nodes of the shaft. Each spring has a stiffness of $10^{8} \mathrm{~N} / \mathrm{mm}$ and a mass $M=0.05 \mathrm{~kg}$ is associated with the central node. The bearing and the casing have 1 element through the thickness. The casing has 36 elements along the circumference and 8 elements through its length. The bearing has 3 elements through its length and 20 along its circumference. The elements are 8-node bricks with constant pressure.

We analyze the first revolution of the rotor after the blade loss with an EDMC (first-order accurate) algorithm with a spectral radius equal to $\rho_{\infty}=0.8$. The time step size is computed from an automatic criterion [20,21] and with an accuracy of $10^{-4}$ on the integration error [20,21] and the choice of updating the Hessian matrix is computed from automatic criteria [20,21]. Each time step is computed with a Newton-Raphson scheme (Tolerance $10^{-5}$ ) enhanced by a line-search system [18] (Tolerance $10^{-3}$ ). The contact interactions are simulated with the physically consistent penalty method proposed by Armero and Petöcz [22,23]. Let us note that we adapted this method to normal-discontinuous surfaces [13]. We will compare the results obtained by this EDMC-1 implicit method with the results obtained by the explicit scheme proposed by Chung and Hulbert [24] that also exhibits numerical dissipation (spectral radius at bifurcation $\rho_{\mathrm{b}}=0.4$ ).

Fig. 8 illustrates the configuration after a quarter of revolution. The free blade interacts with the first next (attached) blade. Fig. 9 illustrates the configuration after half a revolution. The free blade remains between the attached blades and the casing and the attached blades bend. The head 

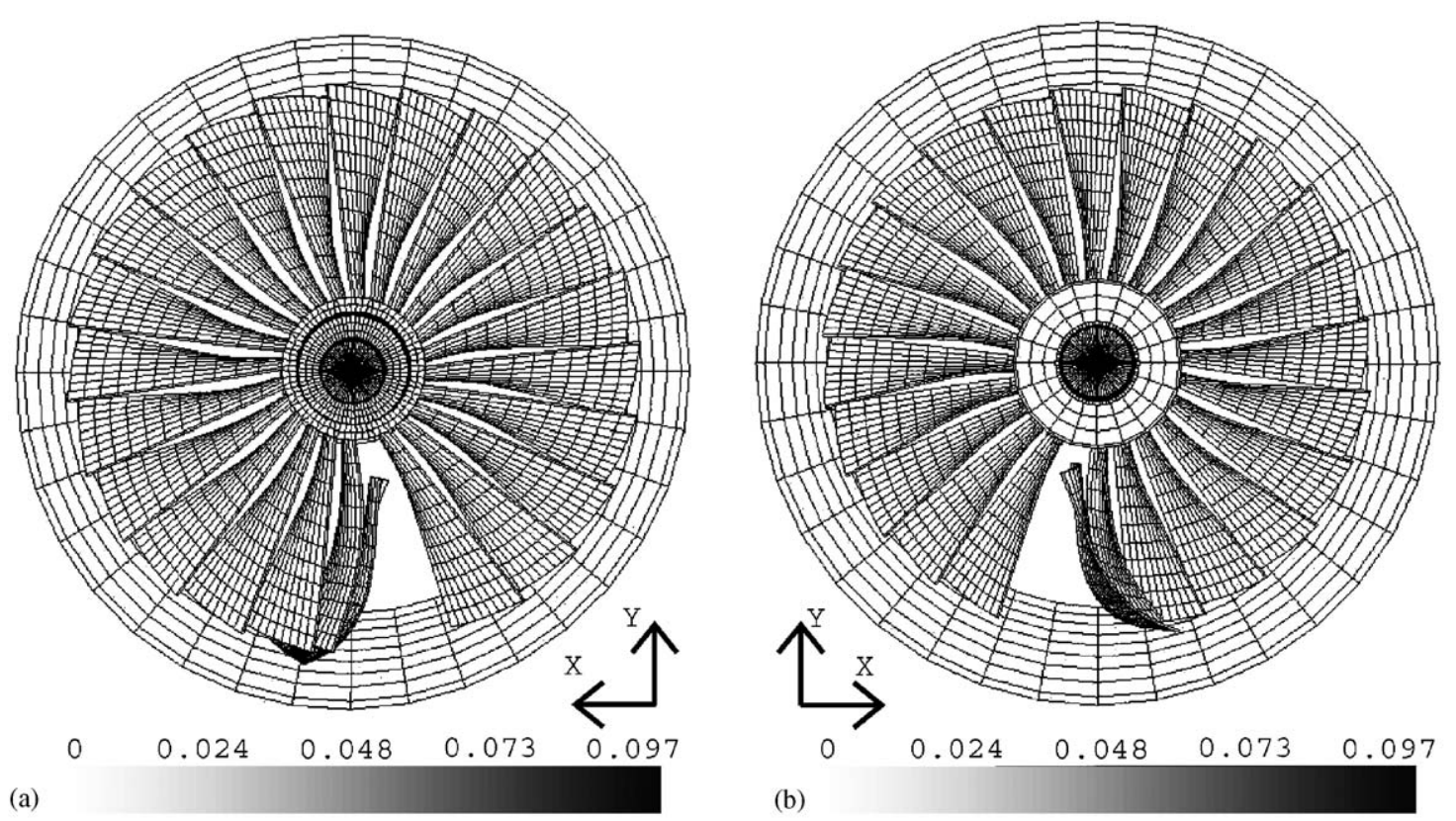

Fig. 8. Configuration and equivalent plastic strain after $\frac{1}{4}$ round.
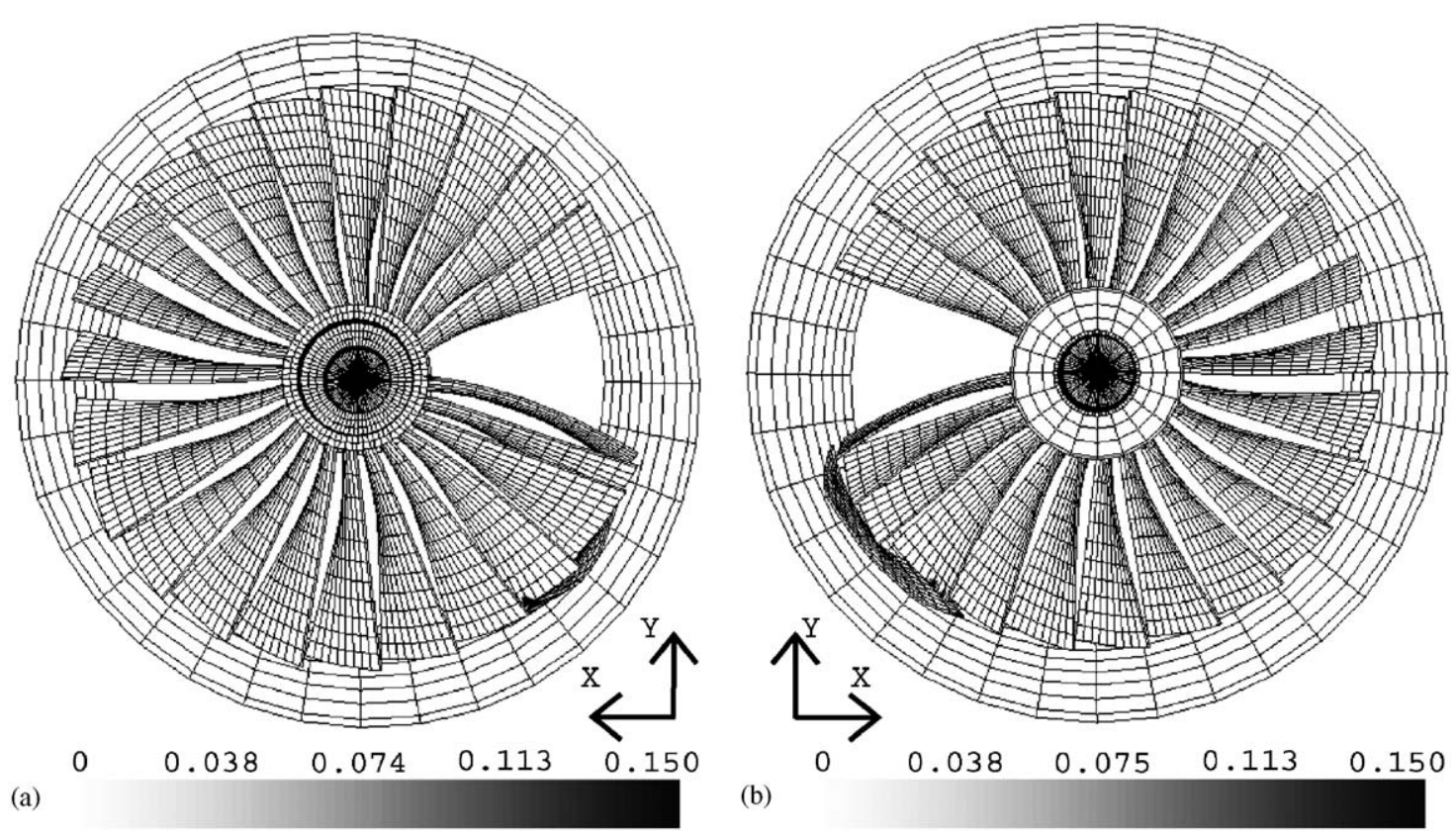

(b)

Fig. 9. Configuration and equivalent plastic strain after $\frac{1}{2}$ round. 


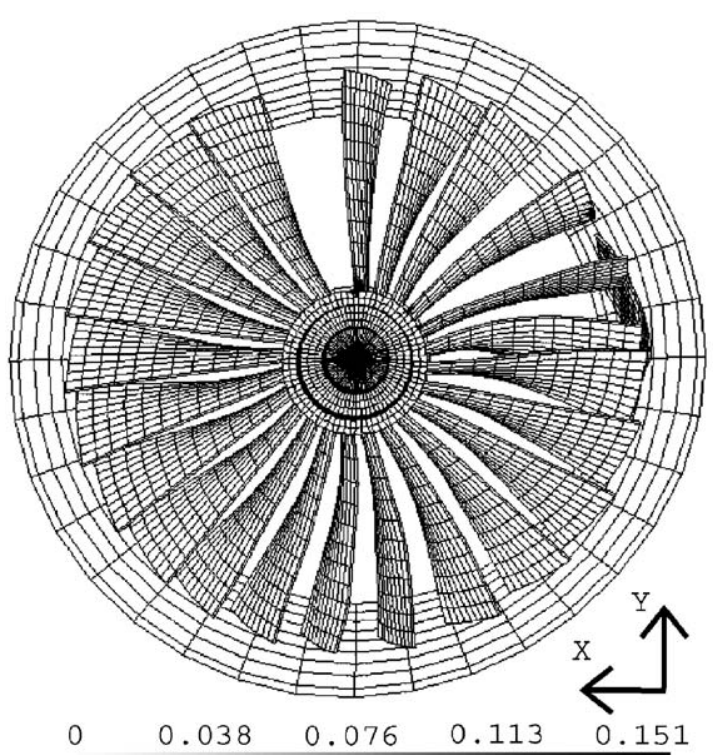

(a)

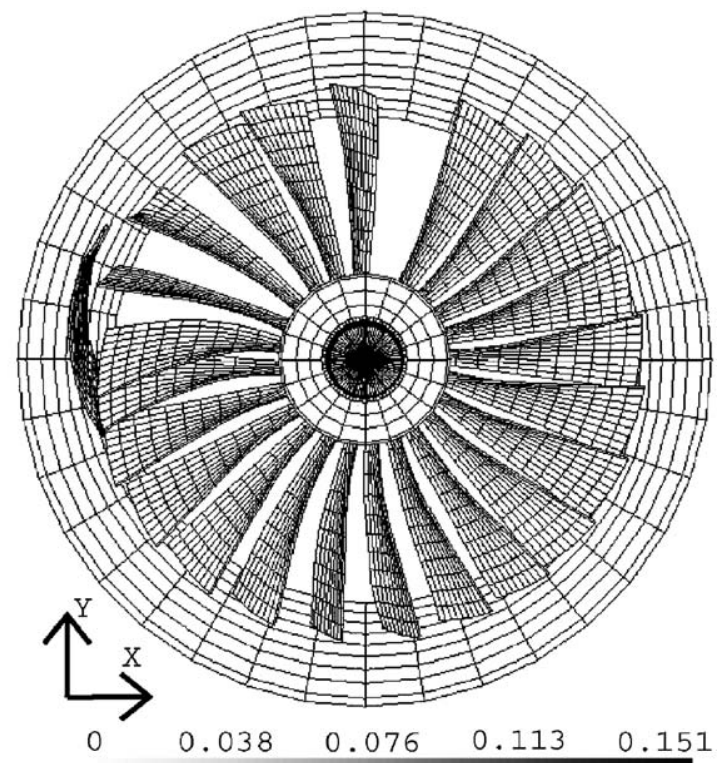

(b)

Fig. 10. Configuration and equivalent plastic strain after $\frac{3}{4}$ round.

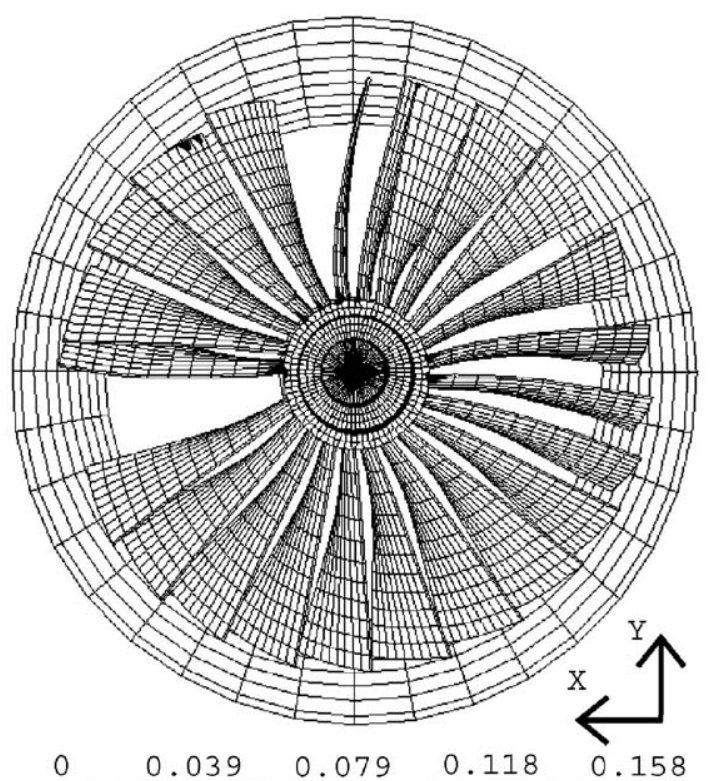

(a)

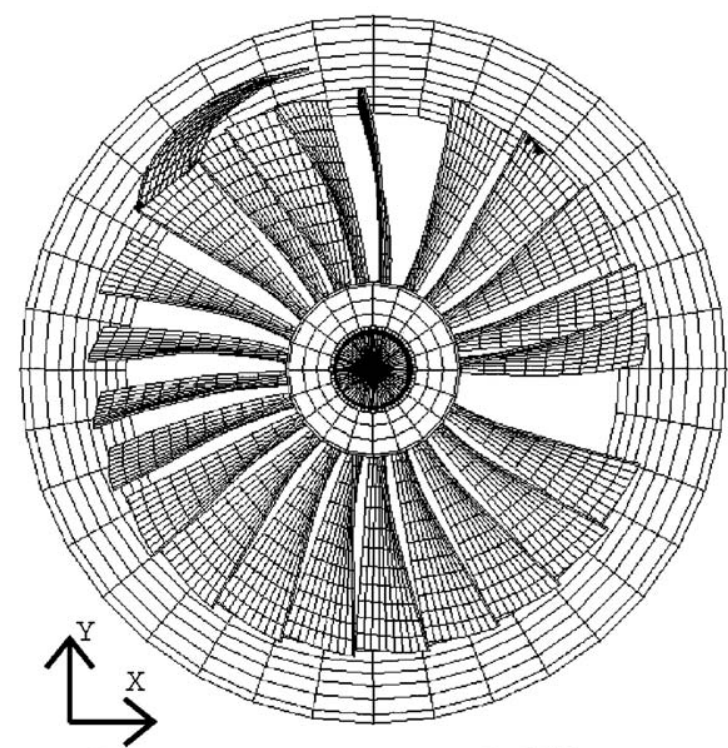

(b)

Fig. 11. Configuration and equivalent plastic strain after 1 round. 


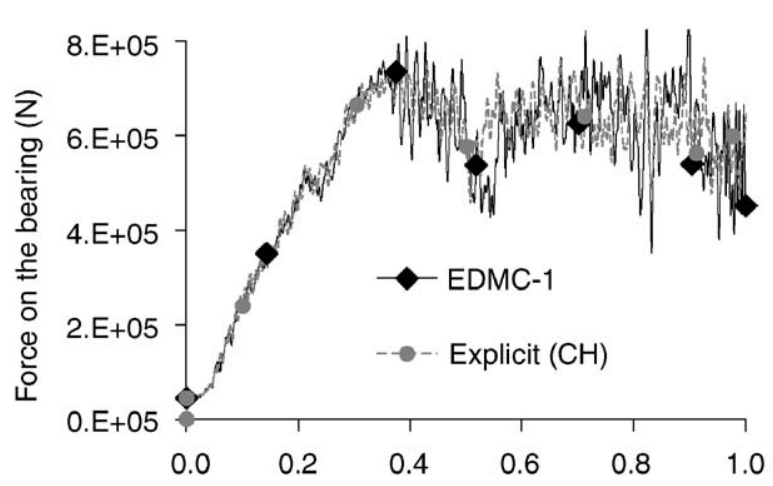

(a)

Round

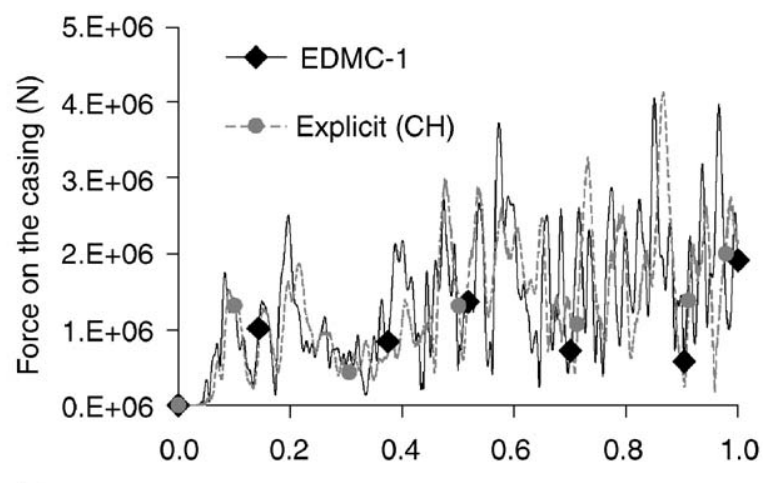

(b)

Round

Fig. 12. Time evolution of the clamping forces.

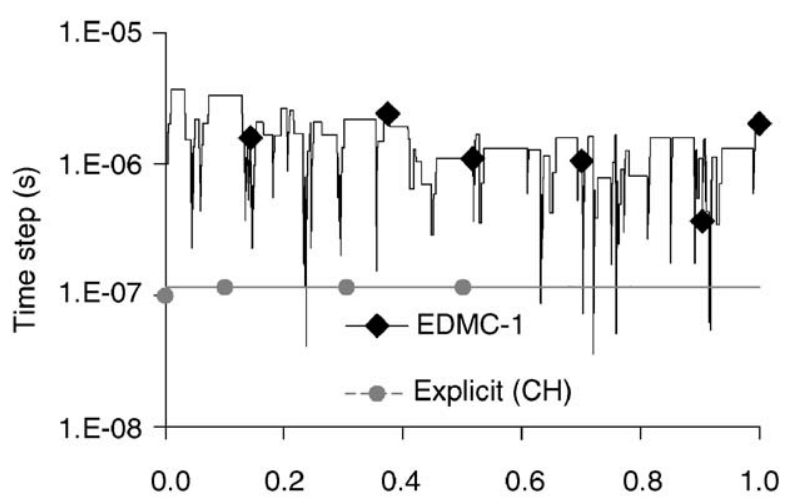

(a)

Round

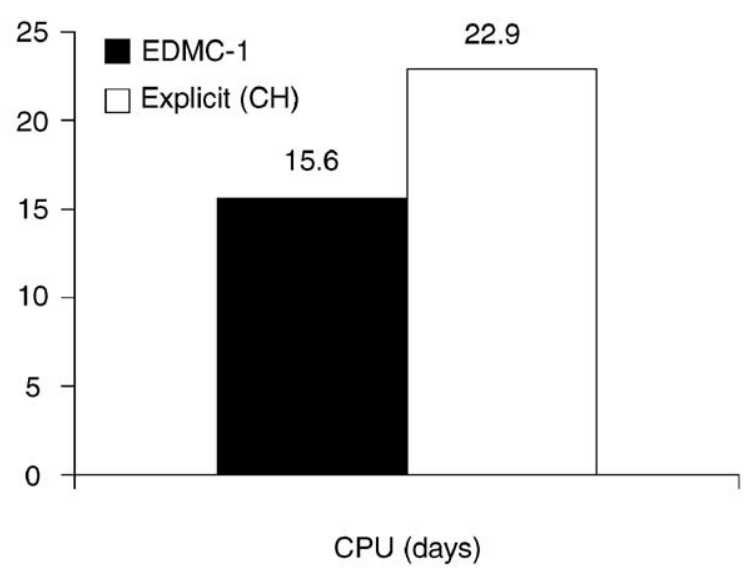

(b)

Fig. 13. Computational cost of the blade loss.

of the free blade enters into contact with the fifth linked blade. Fig. 10 illustrates the deformation after three quarters of a revolution. The head of the free blade has led the fifth blade to bend significantly, and the free blade is pushed towards the rear of the casing. Fig. 11 illustrates the results after one revolution. The free blade was pushed away from the disk so that the remaining interactions only occur between the linked blades and the casing.

The total force on the bearing is illustrated in Fig. 12a. It appears that this force is linear during the first instants, when the bearing reacts to the presence of an unbalanced shaft. But when the free blade interacts with both the linked blades and the casing, the force starts oscillating. Implicit and explicit solutions have the same maximal values. The time evolution of the force on the casing (Fig. 12b) results from the interaction of the blades on the casing and the force oscillates during the whole simulation. Implicit and explicit solutions exhibit the same amplitude peak. This example is an approximation of a real turbo-engine. So we can only compare the implicit solution to the one obtained with an explicit code. But the dynamics is similar to what happens in a real turbo-engine: once the blade is lost it hits the following blades and the casing becomes oval. 
Finally Fig. 13a illustrates the time evolution of the time step size. Most of the time the time step of the implicit method is more than ten times higher than the time step of the explicit method. Since the contact algorithm is very expensive compared to the time needed to solve the explicit scheme, the computational time of the implicit scheme is lower than the computational time of the explicit method (Fig. 13b).

\section{Conclusions}

In this paper we have extended the Energy Dissipative Momentum Conserving algorithm to the elasto-plastic hypoelastic model. We have proved mathematically that this method is stable in the non-linear range and that the numerical dissipation is always positive. We have also performed a spectral analysis in the linear range and have shown that no bifurcation occurs. Next, we have illustrated the accuracy of the proposed method on an academic example. Even for large time step sizes in the non-linear range the stability and the accuracy were preserved although the method is only first-order accurate. By using an automatic time stepping algorithm that ensures accuracy and by considering a physically consistent contact algorithm we were able to present a blade loss problem. This demonstrates the ability of the method to simulate more complex dynamics phenomena. We have compared the results obtained with the results of an explicit simulation. Since the results are similar, we have now an efficient implicit algorithm. Our final purpose is to use this implicit algorithm to achieve an implicit/explicit combination. This combination will be useful when dealing with complex problems.

\section{Appendix. The Jacobian matrix}

To be able to evaluate analytically the Jacobian matrix defined by Eq. (22), we need to known the expression of the dissipation velocities derivative $(\mathbf{G})$ and the expression of the stiffness matrix (K), both defined in Eq. (23).

Let us evaluate tensor $\mathbf{G}\left(\dot{x}^{\mu}\right)$. Eq. (62) leads to

$$
\mathbf{G}(\dot{x})=\frac{\partial}{\partial \dot{\vec{x}}^{n+1}} \vec{G}_{\text {diss }}^{n+1 / 2}=\chi \frac{\left\|\dot{\vec{x}}^{n}\right\|}{\left\|\dot{\vec{x}}^{n+1}\right\|\left[\left\|\dot{\vec{x}}^{n+1}\right\|+\left\|\dot{\vec{x}}^{n}\right\|\right]^{2}}\left[\dot{\vec{x}}^{n+1}+\dot{\vec{x}}^{n}\right] \otimes \dot{\vec{x}}^{n+1}+\frac{\chi}{2} \frac{\left\|\dot{\vec{x}}^{n+1}\right\|-\left\|\dot{\vec{x}}^{n}\right\|}{\left\|\dot{\vec{x}}^{n+1}\right\|+\left\|\dot{\vec{x}}^{n}\right\|} \mathbf{I} .
$$

The stiffness matrix was evaluated for the internal forces in [10]. The part coming from the dissipation forces is evaluated exactly in the same way. Therefore, in this paper we only give the expression that differs. To derivate Eq. (41), one needs to know the derivation of the dissipation $D_{\text {int }}$ defined by Eq. (48). Because of the analogy between the internal forces expressed by Eq. (41) and the dissipation forces expressed by Eq. (51), the only new term to be evaluated is the derivation of the internal dissipation potential expressed by Eq. (54). It leads to

$$
\frac{\partial D_{W}}{\partial\left[\vec{x}^{n+1}\right]^{\mu}}=\frac{\chi}{2} \mathbf{E}_{n}^{\mathrm{e}^{n+1}}: \mathscr{H}: \frac{\partial \mathbf{E}_{n}^{\mathrm{e} \mathrm{e}^{n+1}}}{\partial\left[\vec{x}^{n+1}\right]^{\mu}} J_{0}^{n}+\frac{\chi}{2} \frac{\partial \mathbf{E}_{n}^{\left.\mathrm{e}\right|^{n+1}}}{\partial\left[\vec{x}^{n+1}\right]^{\mu}}: \mathscr{H}: \mathbf{E}_{n}^{\mathrm{e}}{ }^{n+1} J_{0}^{n} .
$$


Let us define $\overline{\mathscr{M}}$ the fourth-order tensor such that

$$
\left\{\mathbf{R}_{n}^{n+1} \frac{\partial\left[\mathscr{H}: \mathbf{E}_{n}^{n+1}-2 G \gamma^{p} \mathbf{N}^{c}\right]}{\partial\left[\vec{x}^{n+1}\right]^{\mu}} \mathbf{R}_{n}^{n+1^{\mathrm{T}}}\right\}_{i j k}=\overline{\mathscr{M}}_{i j k l}\left[\mathbf{f}_{0}^{n+1}\right]_{m l} \vec{D}_{m}^{\mu} .
$$

Its expression can be computed [25,19]: $\overline{\mathscr{M}}_{i j k l}=k \delta_{i j} \delta_{k l}+g^{*} \delta_{i l} \delta_{j k}+g^{*} \delta_{i k} \delta_{j l}-\left(2 g^{*} / 3\right) \delta_{i j} \delta_{k l}-$ $2 g^{*} \mu^{*} \mathbf{N}_{i j} \mathbf{N}_{k l}$ with $g^{*}=\beta G$,

$$
\beta=\sqrt{\frac{2}{3}} \frac{\Sigma^{v, n+1}}{\sqrt{\mathbf{s}^{e}: \mathbf{s}^{e}}}, \quad \mu^{*}=\frac{g^{*}}{1+\frac{h}{3 g^{*}+[\beta-1] h}} \quad \text { and } \quad h=\frac{\partial \Sigma^{v n+1}}{\partial \varepsilon^{p}},
$$

yielding

$$
\frac{\partial D_{W}}{\partial\left[\vec{x}^{n+1}\right]^{\mu}}=\chi\left[\mathbf{R}_{n}^{n+1} \mathbf{E}_{n}^{\mathrm{e}^{n+1}} \mathbf{R}_{n}^{n+1}{ }^{\mathrm{T}}\right]: \overline{\mathscr{M}}\left[\mathbf{f}_{0}^{n+1}\right]^{\mathrm{T}} \vec{D}^{\mu} J_{0}^{n} .
$$

\section{References}

[1] Newmark N. A method of computation for structural dynamics. J Eng Mech Division ASCE 1959;85(EM3): 67-94.

[2] Belytschko T, Schoeberle D. On the unconditional stability of an implicit algorithm for non-linear structural dynamics. J Appl Mech 1975;42:865-9.

[3] Hughes T. Stability, convergence and growth and decay of energy of the average acceleration method in nonlinear structural dynamics. Comput Struct 1976;6:313-24.

[4] Chung J, Hulbert G. A time integration algorithms for structural dynamics with improved numerical dissipations: the generalized- $\alpha$ method. J Appl Mech 1993;60:371-5.

[5] Erlicher S, Bonaventura L, Bursi O. The analysis of the $\alpha$-generalized method for non-linear dynamic problems. Comput Mech 2002;28:83-104.

[6] Simo J, Tarnow N. The discrete energy-momentum method. Conserving algorithms for nonlinear elastodynamics. ZAMP 1992;43:757-92.

[7] Gonzalez O. Exact energy and momentum conserving algorithms for general models in nonlinear elasticity. Comput Methods Appl Mech Eng 2000;190:1763-83.

[8] Meng X, Laursen T. Energy consistent algorithms for dynamic finite deformation plasticity. Comput Methods Appl Mech Eng 2001;191:1639-75.

[9] Noels L, Stainier L, Ponthot J-P. Energy-momentum conserving algorithm for non-linear hypoelastic constitutive models. Int J Numer Methods Eng 2004;59:83-114.

[10] Noels L, Stainier L, Ponthot J-P. On the use of large time steps with an energy-momentum conserving algorithm for non-linear hypoelastic constitutive models. Int J Solids Struct 2004;41:663-93.

[11] Armero F, Romero I. On the formulation of high-frequency dissipative time-stepping algorithms for non-linear dynamics, Part I: low-order methods for two model problems and nonlinear elastodynamics. Comput Methods Appl Mech Eng 2001;190:2603-49.

[12] Armero F, Romero I. On the formulation of high-frequency dissipative time-stepping algorithms for non-linear dynamics, Part II: second-order methods. Comput Methods Appl Mech Eng 2001;190:6783-824.

[13] Noels L, Stainier L, Ponthot J-P. Simulation of crashworthiness problems with improved contact algorithms for implicit time integration. Int J Impact Eng, accepted for publication, doi:10.1016/j.ijimpeng.2005.06.003.

[14] Noels L. Contributions aux algorithmes d'intégration temporelle conservant 1 énergie en dynamique non-linéaire des structures. PhD Thesis. University of Liège, 2004. (in French).

[15] Noels L, Stainier L, Ponthot J-P. Combined implicit/explicit algorithms for crashworthiness analysis. Int J Impact Eng 2004;30:1161-77. 
[16] Noels L, Stainier L, Ponthot J-P. Energy conserving balance of explicit steps to combine implicit and explicit algorithms in structural dynamics. Comput Methods Appl Mech Eng, accepted for publication, doi:10.1016/ j.cma.2005.03.003.

[17] Antman S, editor. Non linear problems of elasticity. Berlin: Springer; 1995.

[18] Crisfield M. Non-linear finite element analysis of solids and structures, vol. 1. New York: Wiley; 2001.

[19] Ponthot J-P. Unified stress update algorithms for the numerical simulation of large deformation elasto-plastic and elasto-viscoplastic processes. Int J Plasticity 2002;18:91-126.

[20] Noels L, Stainier L, Ponthot J-P, Bonini J. Automatic time stepping algorithms for implicit numerical simulations of non-linear dynamics. Adv Eng Software 2002;33(10):581-95.

[21] Noels L, Stainier L, Ponthot J-P. Self-adapting time integration management in crash-worthiness and sheet metal forming computations. Int J Vehicle Design 2002;30(2):67-114.

[22] Armero F, Petöcz E. Formulation and analysis of conserving algorithms for frictionless dynamic contact/impact problems. Comput Methods Appl Mech Eng 1998;158:269-300.

[23] Armero F, Petöcz E. A new dissipative time-stepping algorithm for frictional contact problems: formulation and analysis. Comput Methods Appl Mech Eng 1999;179:151-78.

[24] Hulbert G, Chung J. Explicit time integration algorithms for structural dynamics with optimal numerical dissipation. Comput Methods Appl Mech Eng 1996;137:175-88.

[25] Nagtegaal J, Veldpaus F. On the implementation of finite strain plasticity equations in a numerical model. In: Pittman JFT, et al., editors. Numerical Analysis of Forming Processes. New York: Wiley; 1984. p. 351-71. 